Article

\title{
Preliminary Classification of the ABC Transporter Family in Betula halophila and Expression Patterns in Response to Exogenous Phytohormones and Abiotic Stresses
}

\author{
Linjun An ${ }^{1} \oplus$, Qing Ma ${ }^{1}$, Jinxia Du ${ }^{2}$, Miao Yu ${ }^{1}$, Fangrui Li ${ }^{1}$, Jiayu Luan ${ }^{1}$, Jing Jiang ${ }^{1, *}$ and \\ Huiyu Li ${ }^{1, *}$ \\ 1 State Key Laboratory of Tree Genetics and Breeding, Northeast Forestry University, Harbin 150040, China \\ 2 Changchun Vocational Institute of Technology, Changchun 130033, China \\ * Correspondence: jiangjing1960@126.com (J.J.); lihuiyu2017@126.com (H.L.)
}

Received: 4 July 2019; Accepted: 19 August 2019; Published: 22 August 2019

check for updates

\begin{abstract}
ATP-binding cassette $(\mathrm{ABC})$ transporters comprise a transport system superfamily which is ubiquitous in eukaryotic and prokaryotic cells. In plants, $\mathrm{ABC}$ transporters play important roles in hormone transport and stress tolerance. In this study, $15 \mathrm{BhABC}$ transporters encoded by genes identified from the transcriptome of Betula halophila were categorized into four subfamilies ( $\mathrm{ABCB}, \mathrm{ABCF}, \mathrm{ABCG}$, and $\mathrm{ABCI}$ ) using structural domain and phylogenetic analyses. Upon B. halophila exposure to exogenous phytohormones and abiotic stressors, gene expression patterns and transcriptional responses for each subfamily of genes were obtained using semi-quantitative RT-PCR analysis. The results demonstrated that expression of most genes belonging to $A B C B$ and $A B C G$ subfamilies changed in response to exogenous phytohormone exposures and abiotic stress. These results suggest that $B h A B C$ genes may participate in hormone transport and that their expression may be influenced by ABA-dependent signaling pathways involved in abiotic stress responses to various stressors.
\end{abstract}

Keywords: Betula halophila; ATP-binding cassette transporter; hormone response; abiotic stress response; gene expression

\section{Introduction}

ATP-binding cassette $(\mathrm{ABC})$ transporters are complex proteins that are responsible for the translocation of substrates across membranes. They constitute one of the largest protein families yet discovered, with widespread distribution among eukaryotes and prokaryotes [1,2]. ABC transporters contain a common core structure of nucleotide-binding domains (NBDs) and transmembrane domains (TMDs) [3]. In plants, $\mathrm{ABC}$ transporters can be classified into full transporter, half transporter, and soluble $\mathrm{ABC}$ protein subgroups based on arrangements of these domains [4]. Generally, full transporters contain two NBDs and two TMDs, half transporters contain one NBD and one TMD, and soluble ABC proteins only contain one NBD [4]. According to phylogenetic analyses, $A B C$ transporters can also be classified into eight subfamilies based on evolutionary relatedness (A-G and ABCI; ABCH is not found in plants) $[5,6]$.

In 1992, the first plant ABC transporter gene (AtPGP1) was cloned from Arabidopsis [7]. Since then, the volume of research focusing on the functions of plant $A B C$ transporters has gradually increased and has revealed diverse roles of these proteins. Indeed, the current body of accumulated evidence indicates that these proteins transport plant hormones, metal ions, lipids, secondary metabolites, and exogenous substances, in addition to their roles in regulating plant ion channels and plant-pathogen interactions. Members of the soluble ABC protein subgroup, such as ABCE (Ribonuclease L inhibitor), participate in ribosome recycling and translation termination functions that influence leaf development $[8,9]$. 
To date, research on plant hormone and abiotic stress responses has been predominantly focused on the investigation of these processes in herbs, such as Arabidopsis thaliana.

Using a salt stress model, ABCA subfamily transporters have been shown to be downregulated after $\mathrm{NaCl}$ treatment in Arabidopsis [10], whereas ABCB transporters are mainly involved in the transport of plant hormones, although they can also enhance tolerance of plants to heavy metals [11-15]. ABCC transporters participate in cellular detoxification mainly by pumping toxins and heavy metals out of cells or vacuoles. [16-19]. The ABCG subfamily PDR subgroup of full transporter proteins is primarily responsible for managing abiotic stress and is also involved in hormone regulation [20-22], whereas the ABCG subfamily half transporter (WBC) subgroup of proteins is mainly involved in the transport of plant hormones [23-25].

Betula halophila, a member of the Betulaceae family, is found in the Altay region of northern Xinjiang, China, where it grows in salt marshes to an altitude of $1500 \mathrm{~m}$. Due to its strong salt and drought resistance, $B$. halophila is a useful model species for studying plant resistance to abiotic stress. In a previous study, after B. halophila had been exposed to $200 \mathrm{mM} \mathrm{NaCl}$ for $0 \mathrm{~h}$ or $24 \mathrm{~h}$ to simulate salt stress, cDNA libraries were prepared from its leaves. Subsequently, high-throughput sequencing was conducted to identify differentially expressed genes (SRA: SRP146369) [26]. Gene expression profile analysis detected 15 differentially expressed $B h A B C$ genes in response to salt stress. Based on this result, we studied the expression profiles of these 15 genes in leaves, xylem, roots, and apical buds to better understand $B h A B C$ gene transcriptional responses to exogenous plant hormones and abiotic stressors under various conditions.

\section{Materials and Methods}

\subsection{Plant Materials and Treatment Methods}

B. halophila seeds were collected in Altay, Xinjiang, China and all seeds were half-siblings. After the seeds germinated, they were transplanted into soil within $10 \times 10 \mathrm{~cm}$ culture pots and then cultivated at the Birch Breeding Base of the Northeast Forestry University of Harbin, China. Seedlings were grown for one year and only those with the same growth rate over this period were used in this study.

Seedlings were treated with exogenous hormones and abiotic stress reagents. For hormone treatments, seedlings were sprayed with $25 \mathrm{mg} / \mathrm{L}$ gibberellin $\left(\mathrm{GA}_{3}\right), 1 \mu \mathrm{mol} / \mathrm{L}$ methyl jasmonate (MeJA), $350 \mu \mathrm{mol} / \mathrm{L}$ salicylic acid (SA), $0.2 \mathrm{mg} / \mathrm{L}$ brassinolide (BR), $50 \mathrm{mg} / \mathrm{L}$ cytokinin (6-BA), $100 \mu \mathrm{mol} / \mathrm{L}$ abscisic acid (ABA), or $50 \mathrm{mg} / \mathrm{L}$ auxin (IAA), respectively. For samples undergoing abiotic stress, $20 \%$ Polyethylene Glycol (PEG), $0.4 \mathrm{~mol} / \mathrm{L}$ sodium chloride $(\mathrm{NaCl}), 150 \mu \mathrm{mol} / \mathrm{L}$ cadmium chloride $\left(\mathrm{CdCl}_{2}\right)$, and $0.3 \mathrm{~mol} / \mathrm{L}$ sodium bicarbonate $\left(\mathrm{NaHCO}_{3}\right)$ were used for watering, with no additional watering performed over the study period.

Every day at 8:00 a.m., the spraying of hormones and the irrigation of abiotic stress reagents were carried out. Plants were sampled at various time points during abiotic stress and ABA treatments, namely $0 \mathrm{~h}, 6 \mathrm{~h}, 12 \mathrm{~h}, 24 \mathrm{~h}, 48 \mathrm{~h}$, and $72 \mathrm{~h}$. For all other hormone treatments, plants were sampled at $0 \mathrm{~h}, 2 \mathrm{~h}, 4 \mathrm{~h}, 6 \mathrm{~h}, 12 \mathrm{~h}, 24 \mathrm{~h}, 48 \mathrm{~h}$, and $72 \mathrm{~h}$. At each time point, xylem, roots, leaves, and apical buds of at least five seedlings were collected and pooled, immediately frozen in liquid nitrogen, then stored at $-80{ }^{\circ} \mathrm{C}$ for RNA analysis.

\subsection{Experimental Methods}

\subsubsection{Bioinformatics Analysis}

We used the online National Center of Biotechnology Information (NCBI) Conversed Domains tool (http://www.ncbi.nlm.nih.gov/Structure/cdd/wrpsb.cgi) to predict protein open reading frame (ORF) length and conserved structural domains. The ExPASy tool (https://web.expasy.org/protparam/) was used to reveal amino acid sequences of proteins encoded by these genes and to calculate the theoretical isoelectric point of each protein. Subcellular localization was performed using ProtComp v9.0 (http://linux1. 
softberry.com/berry.phtml?group=programs\&subgroup=proloc\&topic=protcompan). Signal peptides were predicted using SignalP (http://www.cbs.dtu.dk/services/SignalP/). To carry out transmembrane structural region prediction for $\mathrm{BhABC}$ transporter proteins, we used the transmembrane structure analysis program TMHMM v2.0 (http://www.cbs.dtu.dk/services/TMHMM/). The phylogenetic tree was drawn using MEGA 5.0 (developer: Pennsylvania State University, State College, PA, USA) based on the following parameters: neighbor-joining tree method, complete deletion, and 500 bootstrap replicates.

\subsubsection{Semi-Quantitative RT-PCR}

For RNA extraction, we used an RNA extraction kit produced by Universal Plant Total RNA Extraction Kit (BioTeKe, Beijing, China). To perform reverse transcription of RNA to produce cDNA, we used a kit (Rever Tra AceßqPCR RT Master Mix with gDNA Remover, TOYOBO, Shanghai, China). Dilutions of cDNA were used as templates for semi-quantitative RT-PCR analysis. Upstream and downstream primers were designed based on the specific sequence of each gene, with 18s RNA serving as internal reference gene (Table 1). The reaction system for performing semi-quantitative RT-PCR was as follows: TaKaRa rTaq ${ }^{\mathrm{TM}}$ (5 U/ $\mu \mathrm{L}$ ) (TaKaRa, Beijing, China) $0.2 \mu \mathrm{L}, \mathrm{cDNA} 1.0 \mu \mathrm{L}, 1 \mu \mathrm{L}$ of each upstream and downstream primer $(10 \mu \mathrm{mol} / \mathrm{L})$, dNTP mixture ( $2.5 \mathrm{mmol}$ each) $1.6 \mu \mathrm{L}$, and $10 \times$ PCR buffer $\left(\mathrm{Mg}^{2+}\right.$ free) $3.2 \mu \mathrm{L}$. Sufficient $\mathrm{ddH}_{2} \mathrm{O}$ was then added to each sample to adjust the total volume to $20 \mu \mathrm{L}$. Semi-quantitative RT-PCR was conducted using thermal cycling at $94{ }^{\circ} \mathrm{C}$ for $30 \mathrm{~s}, 58^{\circ} \mathrm{C}$ for $30 \mathrm{~s}$, and $72{ }^{\circ} \mathrm{C}$ for $30 \mathrm{~s}$. Amplification was repeated for a total of 35 cycles. The volume of each reaction product solution was adjusted to achieve a consistent concentration of internal reference gene PCR product across samples (to represent a constant number of original plant cells per final adjusted unit volume). In this way, variations in fluorescence intensity of $B h A B C$ PCR product bands, as detected during gel electrophoresis, would reflect differences in $B h A B C$ mRNA quantity in the original cells instead of differences due to variations in sample loading among gel lanes. Each standardized PCR product solution was then electrophoresed on 1\% agarose gels and the intensity of each amplified fragment on the electropherogram was analyzed using Tanon Gis software (developer: Tanon, Shanghai, China) to generate a number representing the relative expression level of a given gene. Finally, heat maps were generated using HemI v1.0 software (developer: The CUCKOO Workgroup, China) based on the following parameter selections: linear normalized intensity data, average linkage clustering method, and similarity metric of Euclidean distance.

Table 1. Primer sequences of $B h A B C 1-B h A B C 15$ genes and internal reference gene 18s RNA.

\begin{tabular}{cccc}
\hline Gene & Upstream Primer Sequence $\left(\mathbf{5}^{\prime} \rightarrow \mathbf{3}^{\prime} \mathbf{)}\right.$ & Downstream Primer Sequence $\left.\mathbf{( 5}^{\prime} \rightarrow \mathbf{3}^{\prime}\right)$ & Length of Product \\
\hline$B h A B C 1$ & CTTACCGGTACATACCTGTAGG & GTATCATGGTGCATTGTTGTCC & $295 \mathrm{bp}$ \\
$B h A B C 2$ & GGTGGTGGACGTAGAAGATCC & GGAAGCTGGAAGAGCATGCC & $297 \mathrm{bp}$ \\
$B h A B C 3$ & TCTCATTCGCTTACGTGTCC & GACCTTACTGAGCAGCTTCC & $289 \mathrm{bp}$ \\
$B h A B C 4$ & CCTCTACCTCCAGGATTAATGC & GCATCACTACCTGATGGTCTGG & $282 \mathrm{bp}$ \\
$B h A B C 5$ & GGATGTTAGCTATCGACCTCC & TGCCACAAAGAACCTCTCAGG & $274 \mathrm{bp}$ \\
$B h A B C 6$ & GAATGGATGAGCAGCAAGTTCC & AACCATCCACAATGAGCTCACG & $279 \mathrm{bp}$ \\
$B h A B C 7$ & AAAGGCAATGCAGAAGCAGG & CTGGTGTCATTTTGGCTACTGC & $251 \mathrm{bp}$ \\
$B h A B C 8$ & CGAACAAAGAAGCTTCGAAGC & CCACATCTCTTCCTCCTACC & $273 \mathrm{bp}$ \\
$B h A B C 9$ & GGATGTTGGAAGTGACTACAGC & GAACTCTATCACGAGCTGTCC & $272 \mathrm{bp}$ \\
$B h A B C 10$ & CATAAGCAGTTCCAACACCTGG & CGGTCCTTGAGCTTTAACAGG & $276 \mathrm{bp}$ \\
$B h A B C 11$ & GCATGTTGGATGGTCACTGG & TAGCATGACGAGAGCAAGAAGC & $261 \mathrm{bp}$ \\
$B h A B C 12$ & TCGGTATGGAGGAACAATGC & GCAATTCCAACTCTGTCGAGG & $290 \mathrm{bp}$ \\
$B h A B C 13$ & GAGAACCGTGCATCATTCTGG & TTCTTCTTCTCGTCTTGTCTGC & $254 \mathrm{bp}$ \\
$B h A B C 14$ & GAATCTCGATTGGAATGGTGC & GGTCAACCTTGTGGTCTTCG & $260 \mathrm{bp}$ \\
$B h A B C 15$ & GGCTGCTTTAGAGAGACTGC & GTAGGCAATGCTCTGCTTCC & $285 \mathrm{bp}$ \\
$18 s r R N A$ & ATCTTGGGTTGGGCAGATCG & CATTACTCCGATCCCGAAGG & $265 \mathrm{bp}$ \\
\hline
\end{tabular}

\section{Results}

\subsection{Sequence Analysis of BhABC Genes in B. halophila}

\subsubsection{Analysis of Physicochemical Properties of BhABC Genes}

We analyzed basic characteristics including open reading frame (ORF) length, amino acid number, isoelectric point, subcellular localization, signal peptide, and transmembrane structure of proteins 
predicted from $15 \mathrm{BhABC}$ genes predicted from the $B$. halophila transcriptome (Table 2). ORF lengths within genes encoding BhABC proteins varied greatly, from $786 \mathrm{bp}$ (BhABC2) to $4296 \mathrm{bp}$ (BhABC12). Isoelectric points of BhABC proteins ranged between 5.41 and 9.68. It was predicted that only BhABC14 contained a signal peptide. Transporter proteins predicted to be located on the cell membrane were BhABC1, BhABC2, BhABC3, BhABC7, BhABC9, BhABC10, BhABC11, BhABC12, and BhABC14. BhABC15, BhABC4, BhABC6, and BhABC8 were predicted to be localized to chloroplast, whereas $\mathrm{BhABC5}$ and $\mathrm{BhABC} 13$ were predicted to be located within mitochondria and nucleus, respectively. The number of transmembrane helices in BhABC transporters varied greatly. BhABC2, BhABC4, BhABC5, BhABC6, BhABC8, and BhABC13 had no transmembrane helix. By contrast, BhABC1, $\mathrm{Bh} A B C 10$, and $\mathrm{BhABC} 12$ proteins each had 13 transmembrane helices, whereas BhABC9 and BhABC11 had 11 and 10 transmembrane helices, respectively. BhABC15 had seven transmembrane helices, BhABC14 had six, and BhABC3 and BhABC7 each had four.

Table 2. Analysis of physical and chemical properties of BhABC genes in Betula halophila.

\begin{tabular}{|c|c|c|c|c|c|c|}
\hline Name & $\begin{array}{l}\text { Length of } \\
\text { ORF (bp) }\end{array}$ & $\begin{array}{l}\text { Number of } \\
\text { Amino Acids }\end{array}$ & $\begin{array}{l}\text { Isoelectric } \\
\text { Point }\end{array}$ & $\begin{array}{l}\text { Subcellular } \\
\text { Localization }^{1}\end{array}$ & $\begin{array}{l}\text { Signal } \\
\text { Peptide }\end{array}$ & $\begin{array}{c}\text { Number of } \\
\text { Transmembrane Helix }\end{array}$ \\
\hline$B h A B C 1$ & 4266 & 1421 & 8.54 & PM & $\mathrm{NO}$ & 13 \\
\hline$B h A B C 3$ & 2265 & 754 & 9.34 & PM & NO & 4 \\
\hline$B h A B C 4$ & 789 & 262 & 6.44 & $\mathrm{CM}$ & $\mathrm{NO}$ & 0 \\
\hline$B h A B C 5$ & 837 & 278 & 9.59 & $\mathrm{M}$ & NO & 0 \\
\hline$B h A B C 8$ & 900 & 299 & 5.81 & $\mathrm{CM}$ & $\mathrm{NO}$ & 0 \\
\hline BhABC9 & 3528 & 1175 & 6.97 & PM & NO & 11 \\
\hline$B h A B C 10$ & 4281 & 1426 & 6.65 & PM & NO & 13 \\
\hline$B h A B C 11$ & 3564 & 1187 & 8.27 & PM & $\mathrm{NO}$ & 10 \\
\hline BhABC12 & 4296 & 1431 & 8.57 & PM & $\mathrm{NO}$ & 13 \\
\hline
\end{tabular}

\subsubsection{Analysis of BhABC Protein Domains}

The ABC transporter domain has various histological forms. In this paper, BlastP provided by the National Center of Biotechnology Information (https://blast.ncbi.nlm.nih.gov/Blast.cgi?PAGE=Proteins) was used to predict conserved NBDs of 15 BhABC proteins (see Figure 1). Using numbers of NBDs, lengths of protein molecules, and results of phylogenetic tree analysis, the $15 \mathrm{BhABC}$ proteins were divided into subfamilies (ABCB, ABCG, ABCF, and ABCI). Within these subfamilies, ABCB (MDR) and ABCG (PDR) proteins are full transporters and each contains two NBDs, whereas ABCB (TAP) and ABCG (WBC) proteins are half transporters with one NBD each. ABCF and ABCI subfamily proteins are soluble $\mathrm{ABC}$ proteins containing one NBD domain each.

\subsubsection{Phylogenetic Analysis of BhABC Proteins}

For phylogenetic analysis, a total of $68 \mathrm{ABC}$ transporters were selected, comprising 24 Oryza sativa transporters, 29 Arabidopsis transporters [27], and 15 BhABC transporters. Phylogenetic tree analysis, carried out using MEGA software, sorted the 68 protein sequences into eight subfamilies (ABCA-ABCG and ABCI) unique to plants (see Figure 2). The ABCB subfamily was further divided into MDR, TAP, and ATM subgroups and the ABCG subfamily into PDR and WBC subgroups. Most $\mathrm{BhABC}$ transporters were found within a limited set of subfamilies (with or without subgroup in parentheses) as follows: ABCB (MDR), ABCB (TAP), ABCF, ABCG (PDR), ABCG (WBC), and ABCI. More specifically, BhABC7 and BhABC11 belonged to ABCB (MDR); BhABC3 belonged to ABCB (TAP); BhABC1, BhABC9, BhABC10, BhABC12, and BhABC15 belonged to ABCG (PDR); BhABC14 belonged 
to $\mathrm{ABCG}$ (WBC); and BhABC5 and BhABC6 belonged to subfamily $\mathrm{ABCF}$. BhABC2, BhABC4, BhABC8, and $\mathrm{BhABC13}$ belonged to subfamily $\mathrm{ABCI}$.

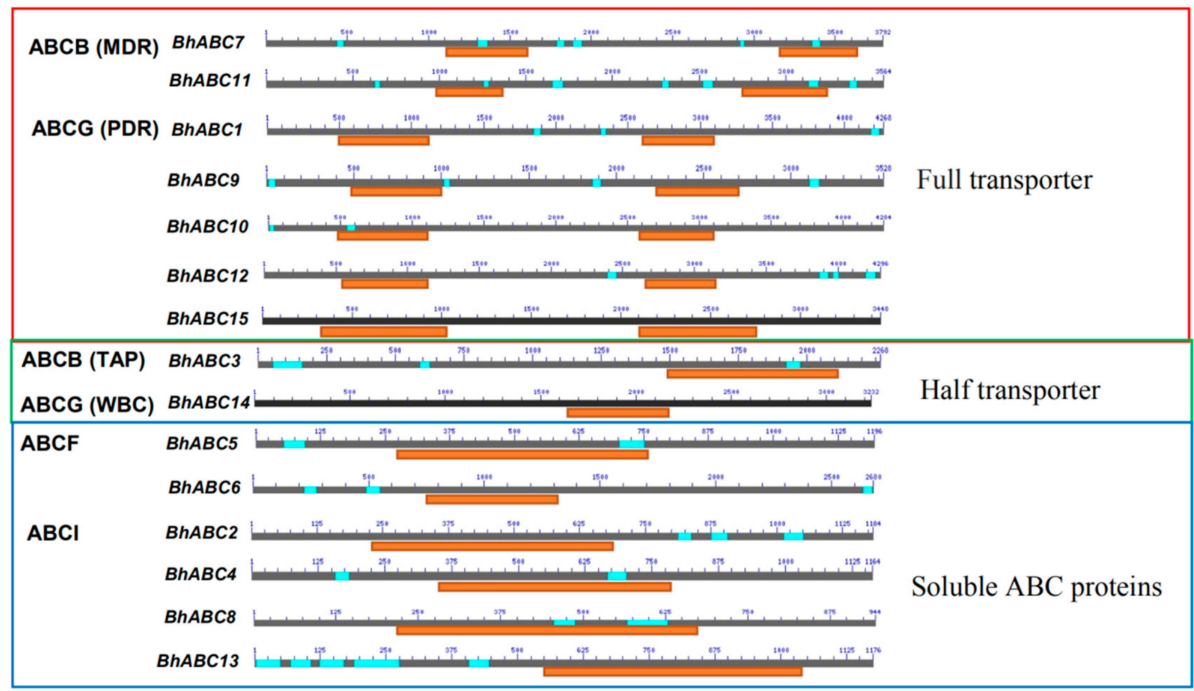

Figure 1. Prediction of conserved domains of $B h A B C$ genes. Gray areas represent exons, blue areas represent introns, and orange areas represent locations of nucleotide-binding domains (NBDs).

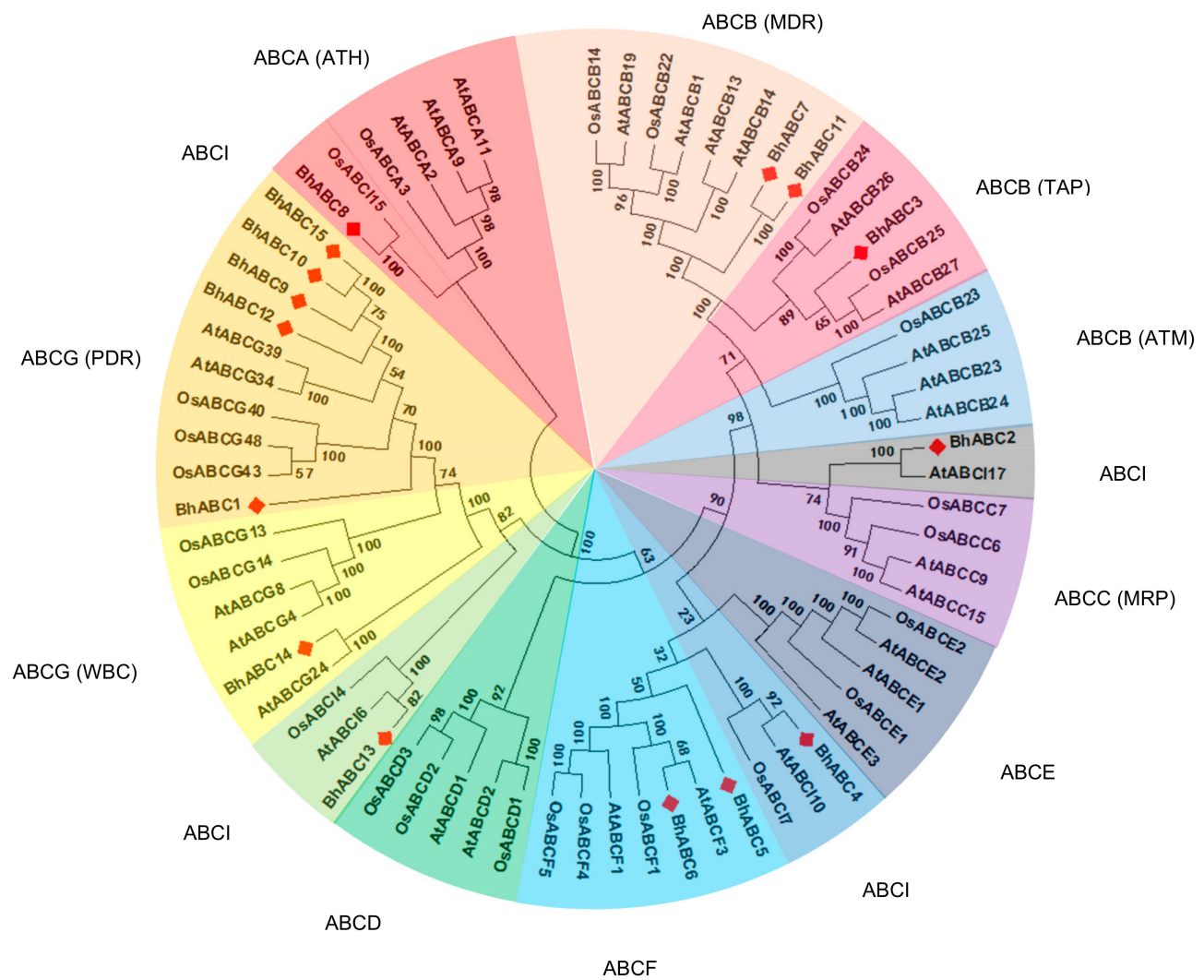

Figure 2. Phylogenetic tree of ABC transporter proteins (BhABC transporters noted with red/orange diamonds). Phylogenetic subclasses are marked with different colors and corresponding subfamily names. The phylogenetic tree was drawn using MEGA 5.0 and was based on the following parameters: neighbor-joining tree method, complete deletion, and 500 bootstrap replicates. OsABC denotes Oryza sativa $\mathrm{ABC}$ proteins (including 24 members), AtABC denotes Arabidopsis ABC proteins (including 29 members), and $\mathrm{Bh} A B C$ denotes $B$. halophila $\mathrm{ABC}$ proteins (including 15 members). Numeric values indicate estimated evolutionary distances. 


\subsection{Responses of BhABC Genes to Hormone Treatments}

\subsubsection{Responses of $B h A B C$ Genes to 6-Benzylaminopurine (6-BA)}

Under 6-BA treatment, altered mRNA expression of most $B h A B C$ genes encoding full transporters and half transporters was observed in xylem, roots, and leaves, whereas in apical buds only the expression of $B h A B C$ genes encoding full transporters changed in response to treatment (see Figure 3). In xylem, mRNA levels reflecting expression of $B h A B C 1, B h A B C 2, B h A B C 4, B h A B C 7$, and $B h A B C 11$ genes were mainly upregulated at all treatment time points except at $72 \mathrm{~h}$. Meanwhile, mRNA expression of $B h A B C 1$ and $B h A B C 2$ peaked at $6 \mathrm{~h}$ and of $B h A B C 4$ peaked at $24 \mathrm{~h}$. BhABC8, BhABC12, and $B h A B C 13$ genes showed upregulated transcriptional responses after $6 \mathrm{~h}$ of treatment, whereas $B h A B C 15$ exhibited a transcriptional response that was first upregulated then downregulated. $B h A B C 3$, $B h A B C 5, B h A B C 6, B h A B C 9, B h A B C 10$, and $B h A B C 14$ expression levels did not change with treatment. In roots, mRNA expression levels of $B h A B C 4$ and $B h A B C 14$ were transiently upregulated at $6 \mathrm{~h}$ and $48 \mathrm{~h}$, respectively. $B h A B C 1, B h A B C 2, B h A B C 7, B h A B C 8, B h A B C 11$, and $B h A B C 15$ genes were mainly upregulated at time points during the middle stage of treatment. Both $B h A B C 1$ and $B h A B C 2$ exhibited peak expression at $6 \mathrm{~h}$. Expression levels of $B h A B C 12$ and $B h A B C 13$ genes increased during middle and late treatment stages, whereas expression of $B h A B C 3, B h A B C 5, B h A B C 6, B h A B C 9$, and $B h A B C 10$ genes did not change. In leaves, $B h A B C 1, B h A B C 4, B h A B C 7, B h A B C 11, B h A B C 12, B h A B C 13$, and BhABC15 were mainly upregulated after $12 \mathrm{~h}$, but $B h A B C 1$ and $B h A B C 4$ reached peak expression levels only at $72 \mathrm{~h}$ and expression of other genes in leaves remained unchanged. In apical buds, only $B h A B C 1$, $B h A B C 7$, and $B h A B C 15$ responded to 6-BA treatment, with expression of $B h A B C 15$ in bud similar to expression in xylem, such that both were upregulated then downregulated, whereas $B h A B C 1$ and $B h A B C 7$ exhibited upregulation at $12 \mathrm{~h}$ and $4 \mathrm{~h}$ of treatment, respectively.

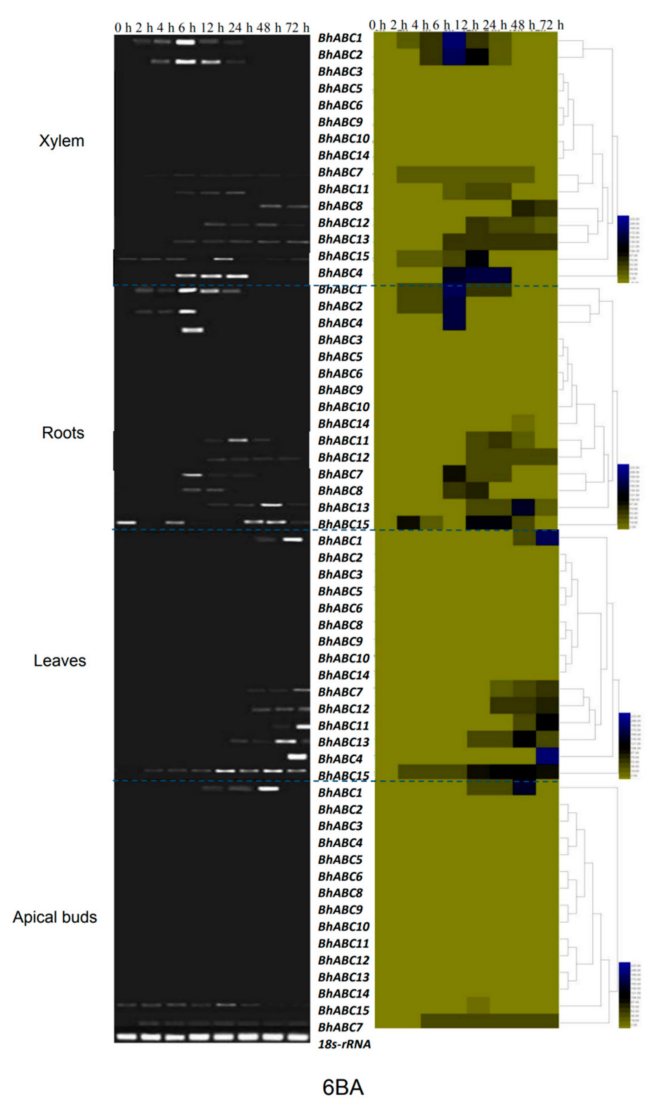

Figure 3. Expression patterns of $B h A B C$ genes in xylem, roots, leaves, and apical buds after 6-BA treatment. Heat map showing responses of $B h A B C$ genes to 6-BA (right); semi-quantitative RT-PCR analysis with 18s RNA internal reference (left). 


\subsubsection{Responses of $B h A B C$ Genes to Brassinolide (BR)}

During BR treatment, $B h A B C$ full transporter genes were the main genes with altered expression in xylem and apical buds, whereas most $B h A B C$ genes among all three subgroups (full transporter, half transporter, soluble $A B C$ proteins) responded to $B R$ in roots and leaves (see Figure 4). In xylem, only $B h A B C 1, B h A B C 11, B h A B C 12, B h A B C 14$, and $B h A B C 15$ responded to $\mathrm{BR}$, with upregulated expression of each after $12 \mathrm{~h}$, whereas expression of $B h A B C 1$ peaked at $48 \mathrm{~h}$. In roots, $B h A B C 15$ was first upregulated then downregulated, $B h A B C 12$ was upregulated early, and $B h A B C 3$ and $B h A B C 10$

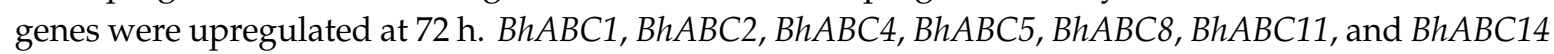
genes all showed different degrees of upregulation in middle and late treatment stages, with $B h A B C 1$ mRNA expression peaking at $72 \mathrm{~h}$. $B h A B C 6, B h A B C 7, B h A B C 9$, and $B h A B C 13$ were unresponsive to $B R$ treatment, with mRNA expression levels remaining unchanged. In leaves, only $B h A B C 3, B h A B C 13$, and $B h A B C 14$ mRNA levels remained unchanged, whereas the remaining genes responded to $B R$ hormone treatment; the expression level of $B h A B C 15$ was initially downregulated then upregulated, whereas other genes were mainly upregulated in the middle and late stages of treatment and $B h A B C 4$

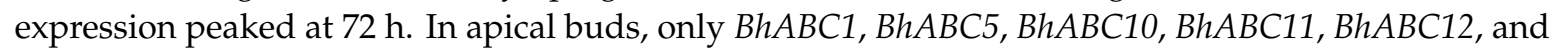
$B h A B C 15$ responded to $B R$, with $B h A B C 15$ mainly showing a downregulated transcriptional response and the other genes exhibiting upregulation during middle and late treatment stages.

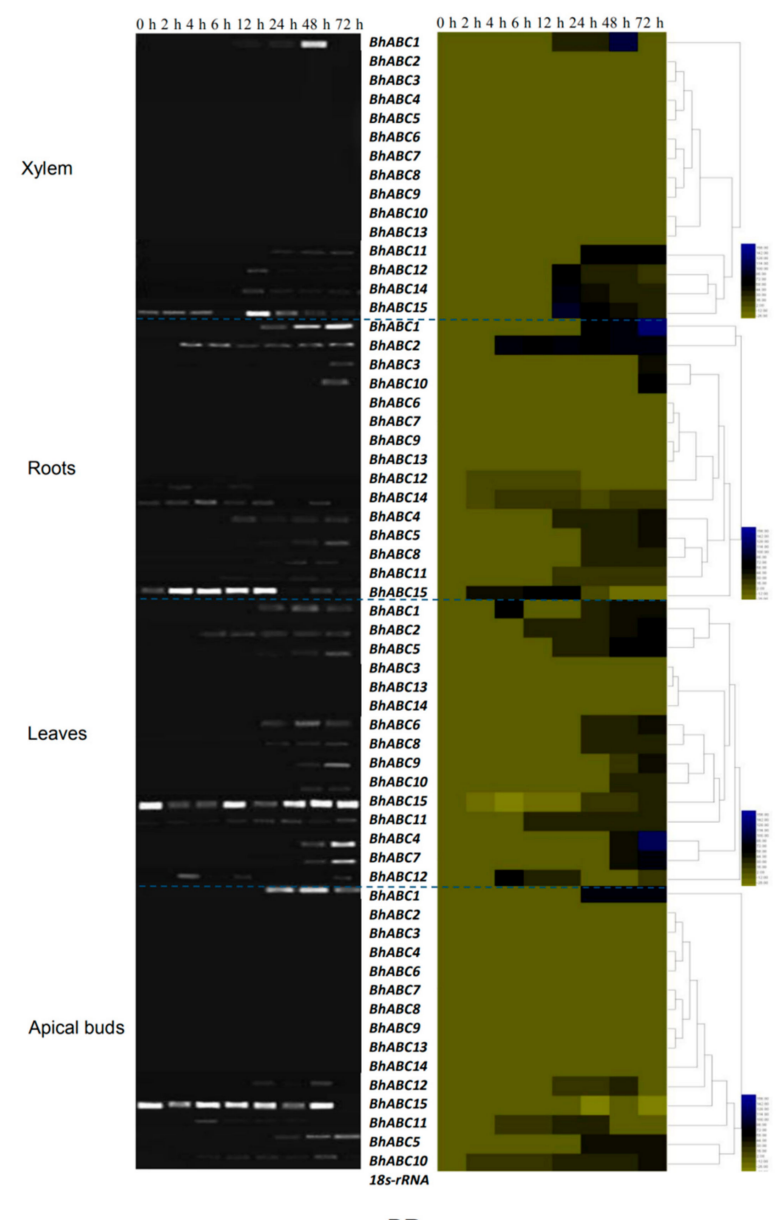

$\mathrm{BR}$

Figure 4. Expression patterns of $B h A B C$ genes in xylem, roots, leaves, and apical buds after BR treatment. Heat map showing responses of $B h A B C$ genes to BR (right); semi-quantitative RT-PCR analysis with 18s RNA internal reference (left). 


\subsubsection{Responses of $B h A B C$ Genes to Gibberellin $\left(\mathrm{GA}_{3}\right)$}

During $\mathrm{GA}_{3}$ treatment (see Figure 5), only $B h A B C 11$, BhABC14, and BhABC15 mRNA expression levels changed in xylem, with downregulation of expression of $B h A B C 11$ and upregulation of $B h A B C 14$ and $B h A B C 15$ observed after $12 \mathrm{~h}$. In roots, mRNA levels of $B h A B C 1, B h A B C 2, B h A B C 3, B h A B C 4$, $B h A B C 5, B h A B C 7, B h A B C 9, B h A B C 10, B h A B C 12$, and $B h A B C 14$ were upregulated after $6 \mathrm{~h}$, with expression of $B h A B C 1$ and $B h A B C 3$ peaking at $48 \mathrm{~h}$. BhABC6, BhABC8,BhABC11, and BhABC13 expression did not change, whereas $B h A B C 15$ was downregulated after $12 \mathrm{~h}$. In leaves, $B h A B C 1$, $B h A B C 2, B h A B C 5$, and $B h A B C 11$ showed upregulated transcriptional responses during the middle stage of treatment, with $B h A B C 1$ and $B h A B C 2$ reaching highest expression levels at $48 \mathrm{~h}$. BhABC10, BhABC12, $B h A B C 13, B h A B C 14$, and $B h A B C 15$ were upregulated during middle and late stages of treatment, whereas $B h A B C 3, B h A B C 4, B h A B C 6, B h A B C 7, B h A B C 8$, and $B h A B C 9$ genes were not expressed. In apical buds, $B h A B C 15$ showed alternate upregulation and downregulation, whereas mRNA levels of $B h A B C 1, B h A B C 5, B h A B C 10, B h A B C 11, B h A B C 12, B h A B C 13$, and $B h A B C 14$ genes were upregulated at most time points throughout the experiment, with expression of $B h A B C 1$ reaching a peak at $48 \mathrm{~h}$. No changes in the expression of remaining genes were observed.

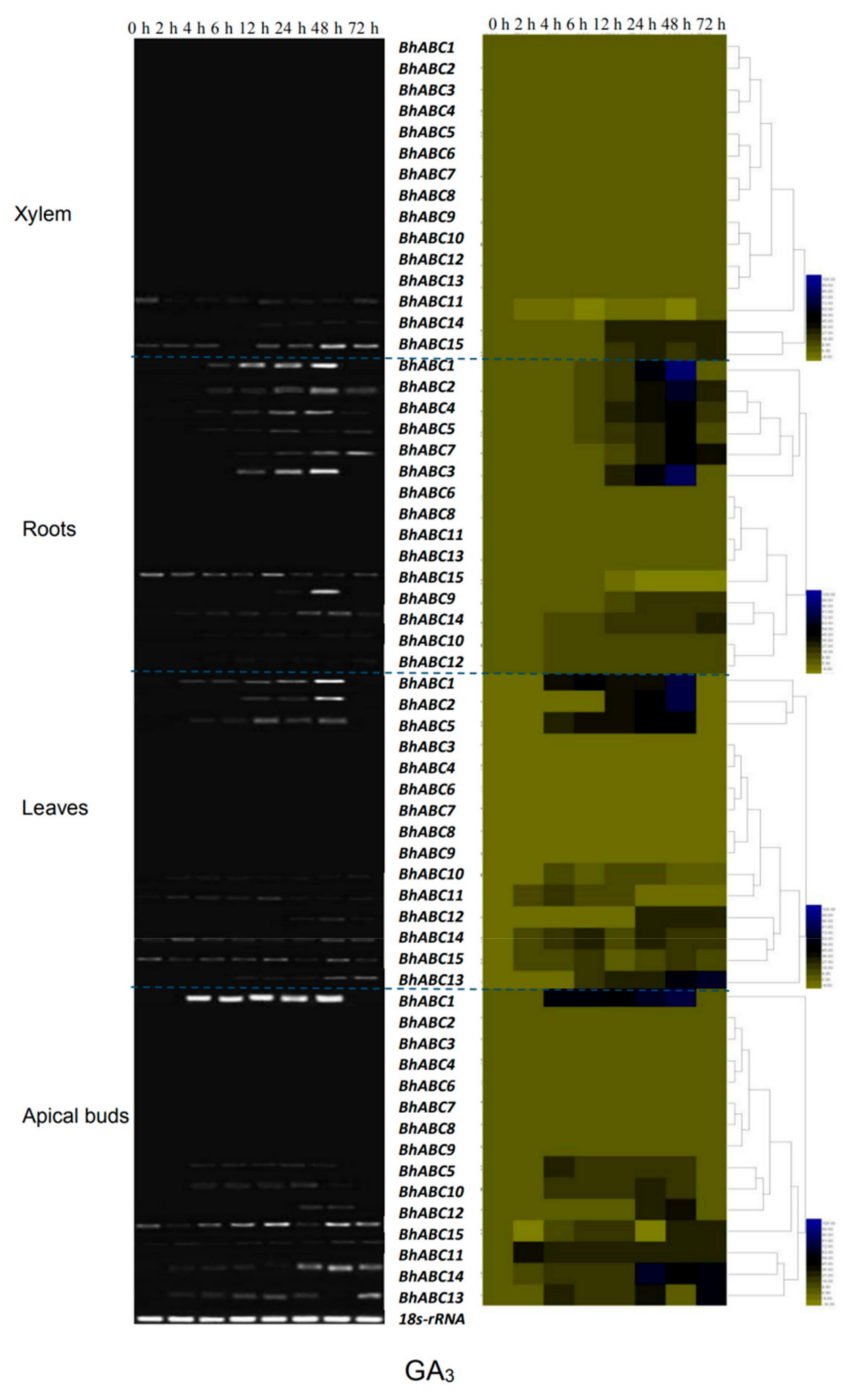

Figure 5. Expression patterns of $B h A B C$ genes in xylem, roots, leaves, and apical buds after $G_{3}$ treatment. Heat map showing responses of $B h A B C$ genes to $\mathrm{GA}_{3}$ (right); semi-quantitative RT-PCR analysis with 18s RNA internal reference (left). 


\subsubsection{Responses of $B h A B C$ Genes to Salicylic Acid (SA)}

During SA treatment, genes encoding three subgroups of two subfamilies of BhABC transporters, namely $A B C G(P D R), A B C G(W B C)$, and $A B C B(M D R)$, exhibited differing degrees of responses among all four tissue sites (see Figure 6). In xylem, mRNA-level expression of $B h A B C 11$ and $B h A B C 15$ was downregulated during early treatment stages, whereas $B h A B C 13$ and $B h A B C 14$ showed upregulated expression after $12 \mathrm{~h}$. No changes in the expression of remaining genes were observed. In roots, only seven genes responded to $\mathrm{SA}$, with expression of $B h A B C 15$ downregulated, whereas $B h A B C 12$ was upregulated at $72 \mathrm{~h}$. Expression levels of $B h A B C 2, B h A B C 3, B h A B C 7, B h A B C 11$, and $B h A B C 14$ peaked in middle and later treatment stages, with mRNA transcription levels of $B h A B C 2$ and $B h A B C 3$ highest at $48 \mathrm{~h}$. In leaves, $B h A B C 14$ and $B h A B C 15$ were downregulated in later treatment stages, $B h A B C 1, B h A B C 2$, $B h A B C 3, B h A B C 5, B h A B C 6, B h A B C 7, B h A B C 10, B h A B C 11$, and $B h A B C 12$ genes were upregulated in middle and later treatment stages, and no changes in expression of $B h A B C 4, B h A B C 8, B h A B C 9$, and $B h A B C 13$ were observed. In apical buds, $B h A B C 14$ exhibited upregulation then downregulation of transcriptional response. Meanwhile, $B h A B C 11$ and $B h A B C 12$ were briefly upregulated between $2 \mathrm{~h}$ and $4 \mathrm{~h}$, whereas $B h A B C 1, B h A B C 6, B h A B C 10$, and $B h A B C 15$ genes were upregulated during middle and later treatment stages, and $B h A B C 15$ expression peaked at $72 \mathrm{~h}$. No changes in the expression of remaining genes were observed in response to SA treatment.

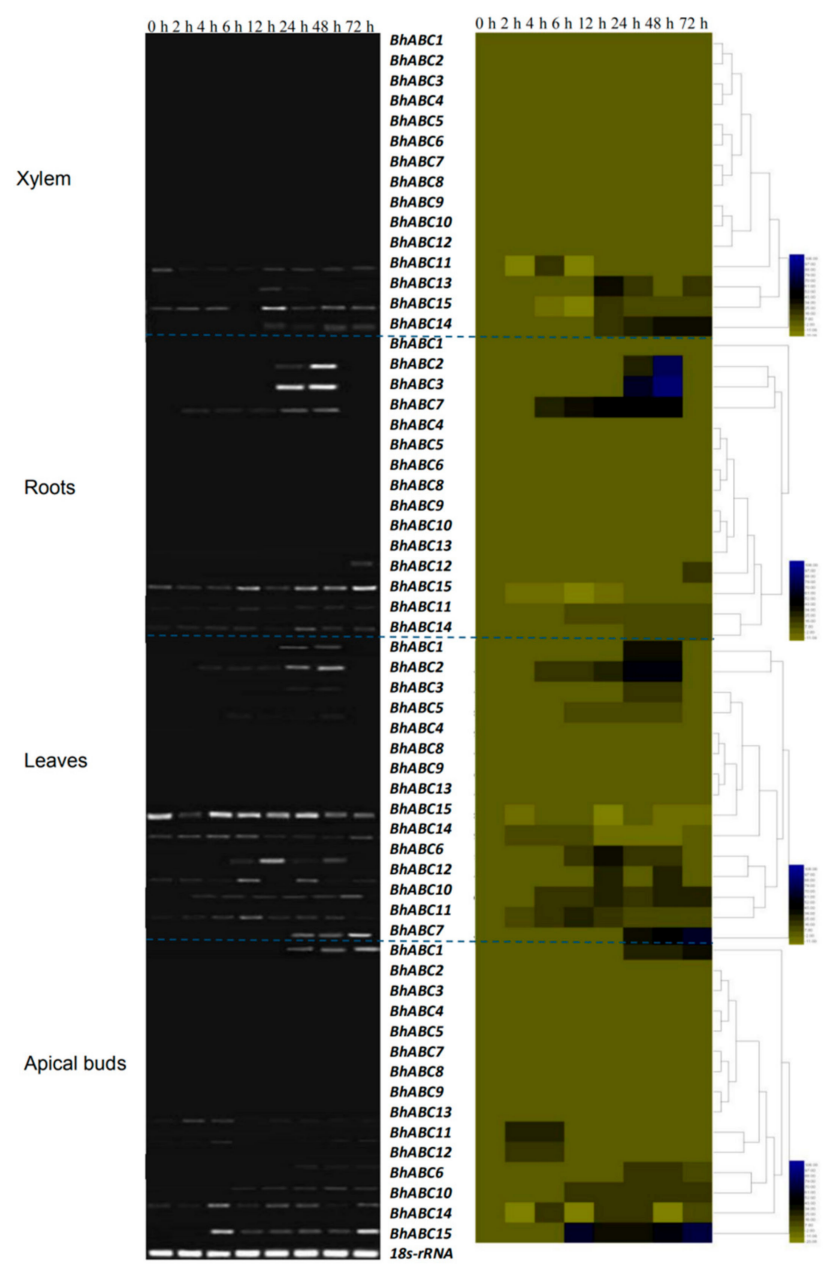

SA

Figure 6. Expression patterns of $B h A B C$ genes in xylem, roots, leaves, and apical buds after SA treatment. Heat map showing responses of $B h A B C$ genes to SA (right); semi-quantitative RT-PCR analysis with 18s RNA internal reference (left). 


\subsubsection{Responses of BhABC Genes to Jasmonic Acid (JA)}

During JA treatment, generally most BhABC full transporter and half transporter gene subgroups

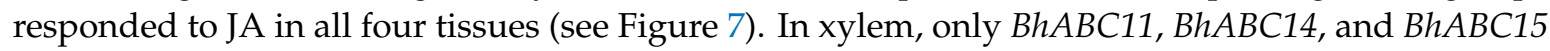
responded to JA, with mRNA expression of $B h A B C 11$ and $B h A B C 14$ upregulated then downregulated during middle and later treatment stages. In roots, five genes responded to JA, including $B h A B C 14$, which was upregulated then downregulated, whereas $B h A B C 9, B h A B C 11, B h A B C 12$, and $B h A B C 15$ were all upregulated and $B h A B C 15$ peaked at $2 \mathrm{~h}$. In leaves, $B h A B C 2, B h A B C 7, B h A B C 9, B h A B C 11$, and $B h A B C 12$ were all upregulated after $24 \mathrm{~h}$. By contrast, expression levels of $B h A B C 14$ and $B h A B C 15$ decreased during early treatment stages and the remaining genes experienced no changes in expression. In apical buds, $B h A B C 15$ exhibited upregulation followed by downregulation, whereas $B h A B C 1$, $B h A B C 2, B h A B C 3, B h A B C 4, B h A B C 6, B h A B C 7, B h A B C 9, B h A B C 11, B h A B C 13$, and BhABC14 were upregulated during middle and late treatment stages. No changes were observed in $B h A B C 5, B h A B C 8$, $B h A B C 10, B h A B C 12$, and BhABC13 expression.

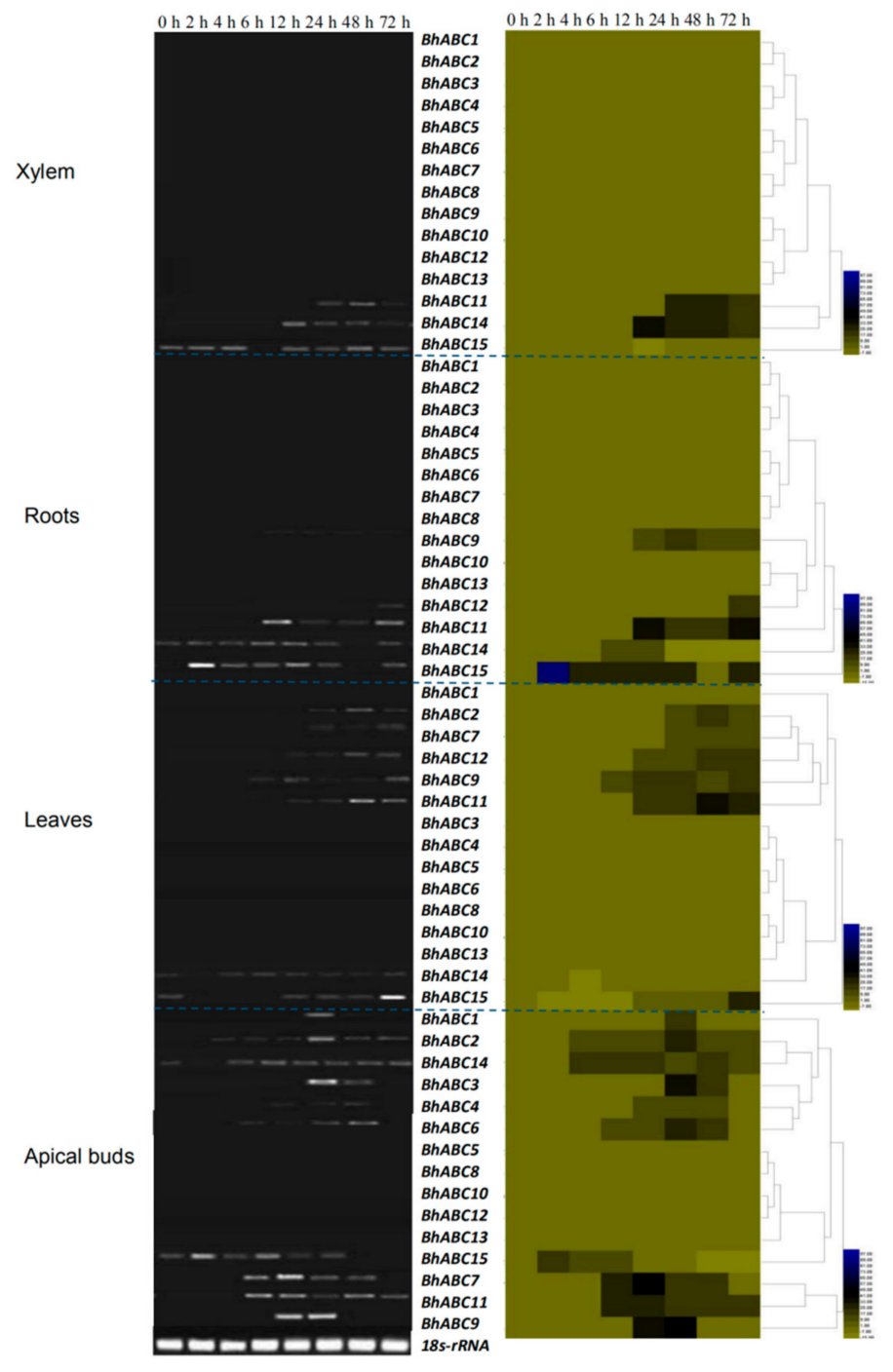

JA

Figure 7. Expression patterns of $B h A B C$ genes in xylem, roots, leaves, and apical buds after JA treatment. Heat map showing responses of $B h A B C$ genes to JA (right); semi-quantitative RT-PCR analysis with $18 \mathrm{~s}$ RNA internal reference (left). 


\subsubsection{Responses of BhABC Genes to Indole-3-Acetic Acid (IAA)}

After IAA treatment (see Figure 8), in xylem and roots $B h A B C$ genes responding to IAA were mainly full and half transporters, whereas in leaf and terminal buds all three types of BhABC proteins responded to IAA. In xylem, only $B h A B C 14$ responded to IAA and exhibited upregulated expression in middle and late treatment stages. In roots, $B h A B C 2, B h A B C 11, B h A B C 14$, and $B h A B C 15$ were upregulated during middle and later stages of IAA treatment, whereas remaining genes were not

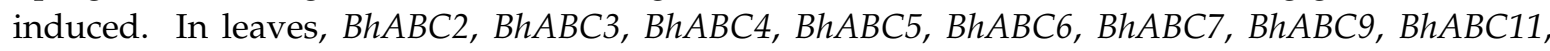
$B h A B C 14$, and $B h A B C 15$ exhibited different degrees of upregulation during middle and late treatment stages, with $B h A B C 11$ peaking at $48 \mathrm{~h}$ and expression of $B h A B C 1, B h A B C 8, B h A B C 10, B h A B C 12$, and $B h A B C 13$ unchanged. Expression patterns of $B h A B C$ genes in apical buds were similar to patterns in leaves, with $B h A B C 11$ expression peaking at $48 \mathrm{~h}$ as well.

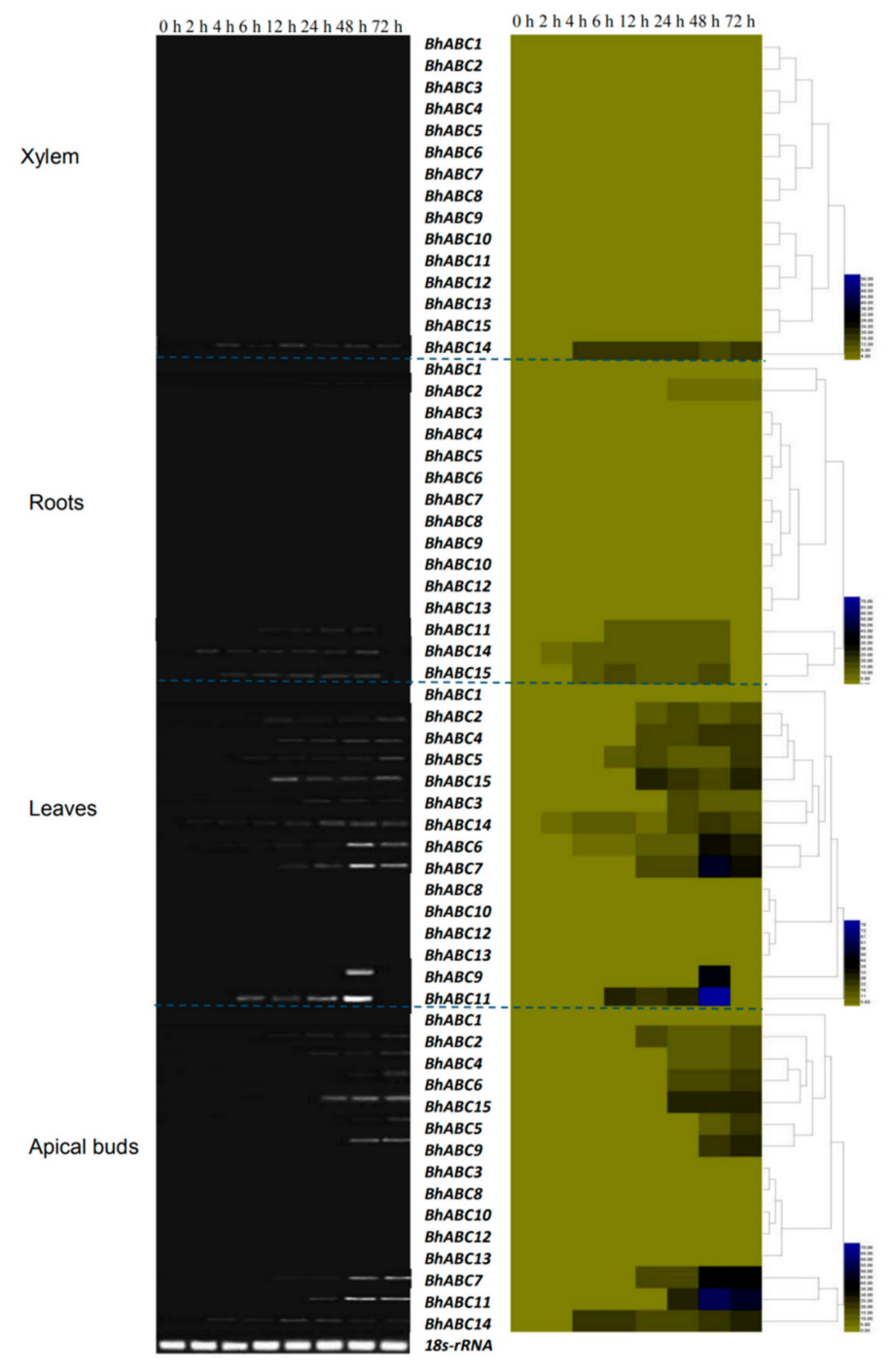

IAA

Figure 8. Expression patterns of $B h A B C$ genes in xylem, roots, leaves, and apical buds after IAA treatment. Heat map showing responses of $B h A B C$ genes to IAA (right); semi-quantitative RT-PCR analysis with 18s RNA internal reference (left).

\subsubsection{Responses of $B h A B C$ Genes to Abscisic Acid (ABA)}

In response to $\mathrm{ABA}$ treatment, expression of the three subgroups of $B h A B C$ genes in all four studied tissues responded to ABA (see Figure 9). In xylem, only $B h A B C 3, B h A B C 11, B h A B C 14$, and 
$B h A B C 15$ showed upregulated expression, which occurred during middle and late treatment stages, with remaining genes not induced. In roots, $B h A B C 1, B h A B C 6, B h A B C 8, B h A B C 11, B h A B C 12, B h A B C 14$, and $B h A B C 15$ were upregulated during middle and late stages of treatment, whereas no other genes were induced. In leaves, $B h A B C 1, B h A B C 2, B h A B C 3, B h A B C 6, B h A B C 8, B h A B C 11, B h A B C 12, B h A B C 14$, and $B h A B C 15$ all exhibited upregulated expression after $48 \mathrm{~h}$, with expression levels of $B h A B C 1$ and $B h A B C 15$ peaking at $72 \mathrm{~h}$ and remaining genes not induced. In apical buds, $B h A B C 4, B h A B C 5$, and $B h A B C 13$ RNA levels were unchanged, but the other genes exhibited upregulated expression to varying degrees during middle and late stages of treatment, with $B h A B C 1, B h A B C 2, B h A B C 3$, and $B h A B C 15$ upregulated to the greatest extent between $48 \mathrm{~h}$ and $72 \mathrm{~h}$.

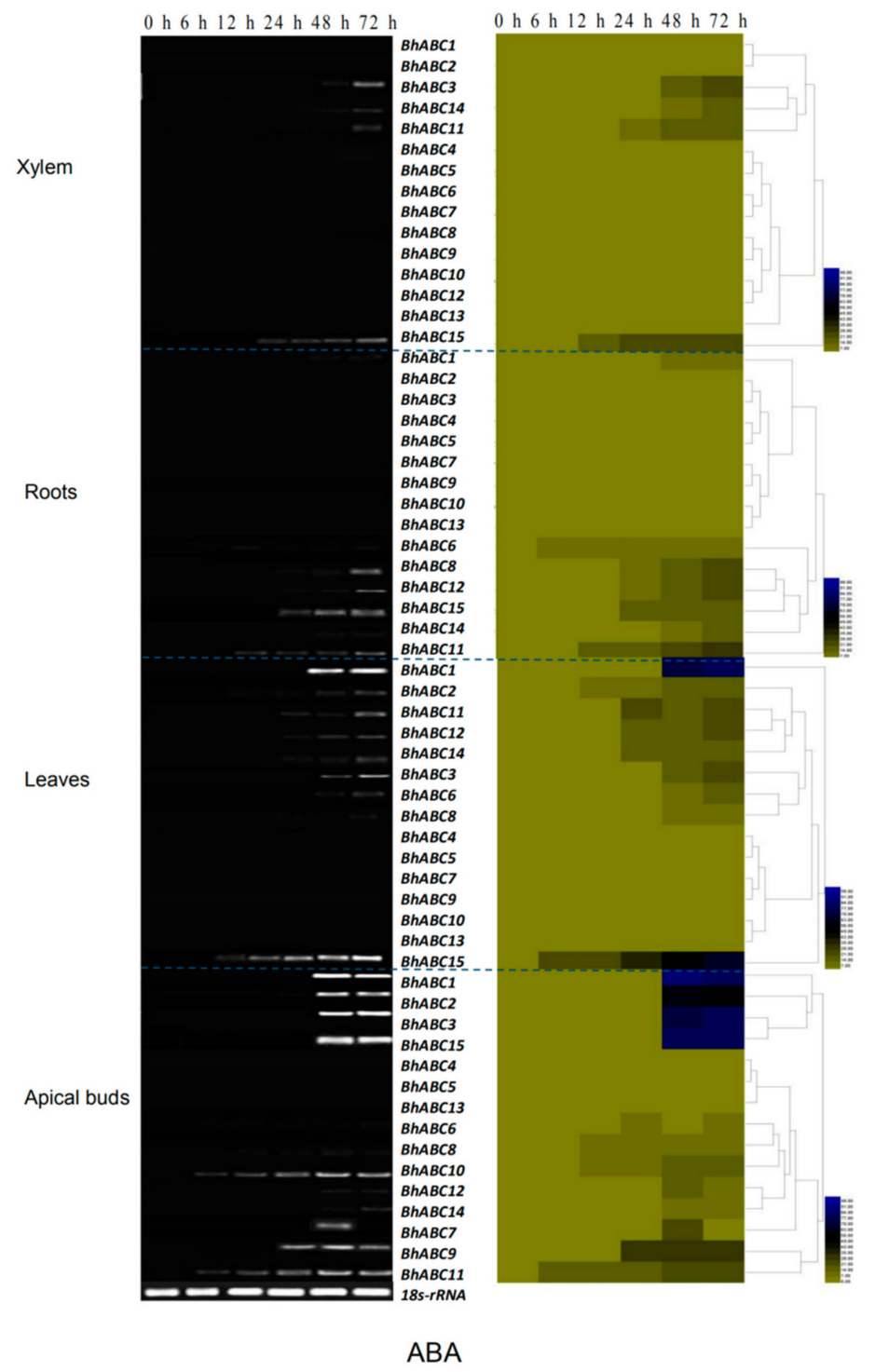

Figure 9. Expression patterns of $B h A B C$ genes in xylem, roots, leaves, and apical buds after $A B A$ treatment. Heat map showing responses of $B h A B C$ genes to ABA (right); semi-quantitative RT-PCR analysis with 18s RNA internal reference (left).

\subsection{Expression Characteristics of BhABC Genes under Abiotic Stress}

\subsubsection{Expression of $B h A B C$ Genes under $\mathrm{NaCl}$ Stress}

Under conditions of $\mathrm{NaCl}$ stress, the three subgroups of BhABC proteins showed variable degrees of responsiveness among all four tissue sites (see Figure 10). In xylem, BhABC15 exhibited 
downregulated expression after $6 \mathrm{~h}$, whereas $B h A B C 2, B h A B C 3, B h A B C 5, B h A B C 6, B h A B C 10, B h A B C 11$, $B h A B C 12, B h A B C 13$, and $B h A B C 14$ all exhibited upregulated expression to varying degrees during middle and later stages of treatment. No changes in expression of $B h A B C 1, B h A B C 4, B h A B C 7, B h A B C 8$, and $B h A B C 9$ were observed. In roots, $B h A B C 15$ exhibited downregulated expression, whereas $B h A B C 2$ and $B h A B C 6$ exhibited upregulated expression at $72 \mathrm{~h}$ and $B h A B C 7$ exhibited a transient increase in expression at $24 \mathrm{~h}$. Meanwhile, $B h A B C 3, B h A B C 5, B h A B C 11, B h A B C 13$, and $B h A B C 14$ showed higher expression levels during middle or later treatment stages, whereas expression of $B h A B C 3$ and $B h A B C 11$ peaked at $24 \mathrm{~h}$. No changes in the expression of remaining genes were observed. In leaves, only $B h A B C 8$ did not respond to $\mathrm{NaCl}$ treatment, whereas $B h A B C 15$ showed upregulation followed by downregulation. All remaining genes were upregulated during middle and late treatment stages, with expression of $B h A B C 1$ and $B h A B C 9$ peaking at $24 \mathrm{~h}$. In apical buds, $B h A B C 3, B h A B C 8$, and $B h A B C 15$ were not induced, whereas all other genes exhibited upregulation during the middle treatment stage.

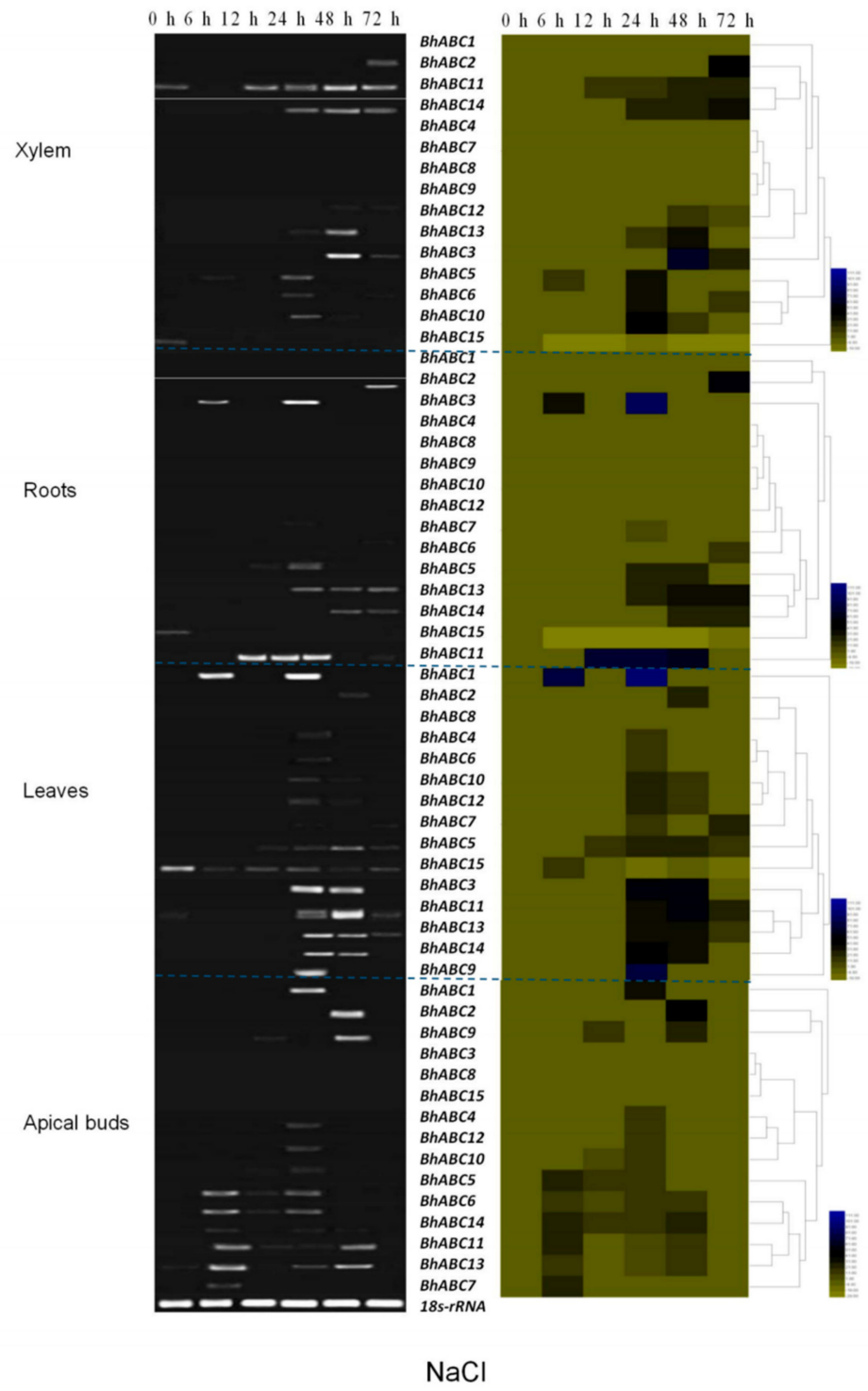

Figure 10. Expression patterns of $B h A B C$ genes in xylem, roots, leaves, and apical buds after $\mathrm{NaCl}$ treatment. Heat map showing responses of $B h A B C$ genes to $\mathrm{NaCl}$ (right); semi-quantitative RT-PCR analysis with 18s RNA internal reference (left). 


\subsubsection{Expression of $B h A B C$ Genes under $\mathrm{CdCl}_{2}$ Stress}

As for $\mathrm{NaCl}$ stress, three subgroups of $\mathrm{BhABC}$ proteins responded to $\mathrm{CdCl}_{2}$ stress in all four tissues (see Figure 11). In xylem, $B h A B C 11$ and $B h A B C 15$ were upregulated then downregulated, whereas $B h A B C 1, B h A B C 2, B h A B C 5, B h A B C 6, B h A B C 8, B h A B C 12$, and $B h A B C 14$ were upregulated during middle and later stages of treatment, with expression of $B h A B C 1, B h A B C 2$, and $B h A B C 6$ genes significantly upregulated at $48 \mathrm{~h}$. In roots, only $B h A B C 7, B h A B C 10$, and $B h A B C 13$ did not respond to $\mathrm{CdCl}_{2}$, whereas $B h A B C 1, B h A B C 3, B h A B C 6$, and $B h A B C 8$ exhibited downregulation. BhABC11, $B h A B C 12$, and $B h A B C 15$ showed upregulation then downregulation, whereas all remaining genes were upregulated to varying degrees. In leaves, all genes except $B h A B C 7$ responded to $\mathrm{CdCl}_{2}$ treatment, with $B h A B C 14$ and $B h A B C 15$ exhibiting upregulation then downregulation and remaining genes exhibiting upregulation to varying degrees during middle and later treatment stages. In apical buds, $B h A B C 10$ was downregulated in the middle treatment stage, whereas BhABC1,BhABC9,BhABC14 , and BhABC15 exhibited upregulation then downregulation. Meanwhile, $B h A B C 3, B h A B C 4, B h A B C 5$, and $B h A B C 6$ were upregulated dramatically at $6 \mathrm{~h}$, with upregulation and high expression of $B h A B C 11$ observed at $72 \mathrm{~h}$. No changes in the expression of remaining genes were observed.

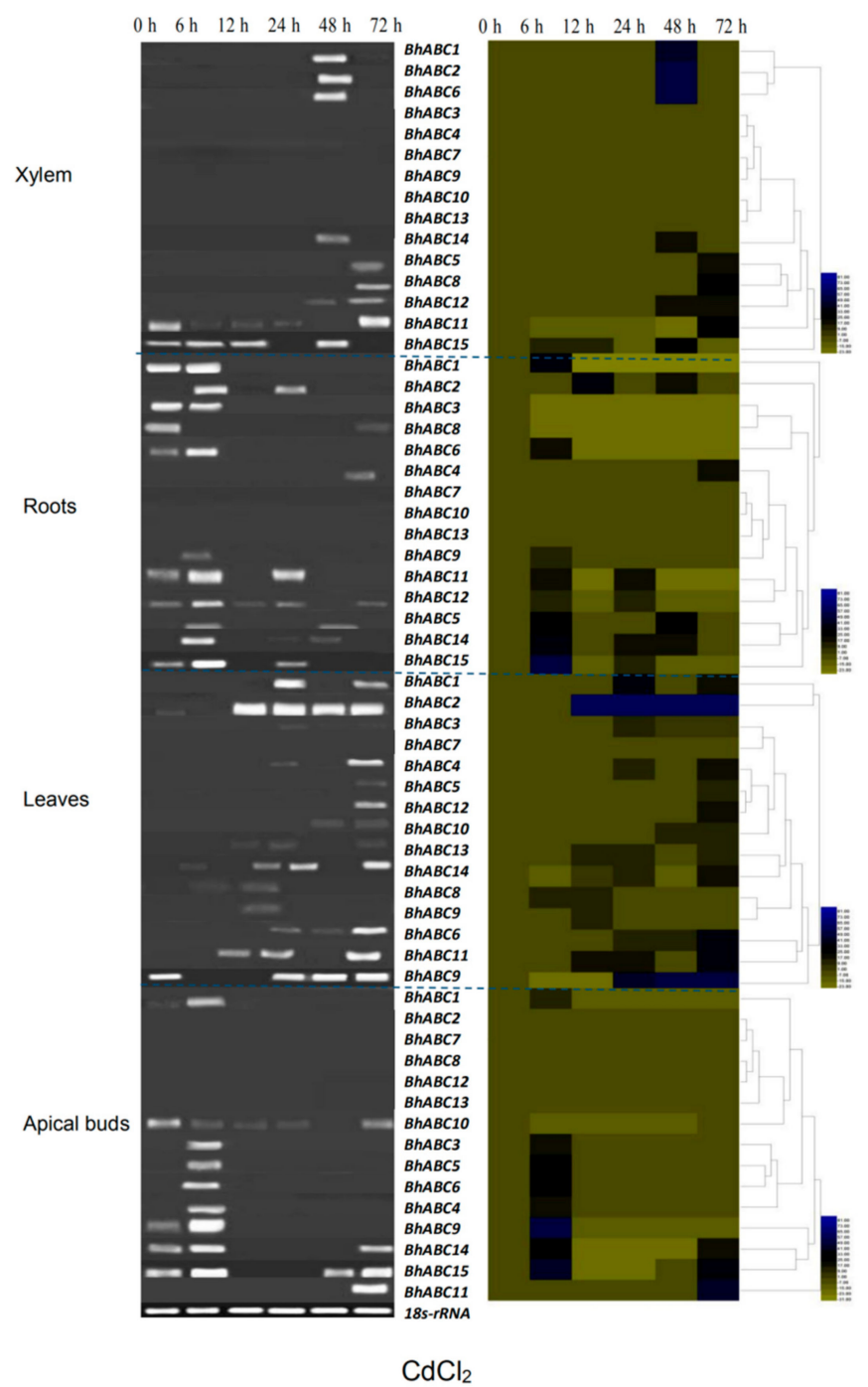

Figure 11. Expression patterns of $B h A B C$ genes in xylem, roots, leaves, and apical buds after $\mathrm{CdCl}_{2}$ treatment. Heat map showing responses of $B h A B C$ genes to $\mathrm{CdCl}_{2}$ (right); semi-quantitative RT-PCR analysis with 18s RNA internal reference (left). 


\subsubsection{Expression of $B h A B C$ Genes under $\mathrm{NaHCO}_{3}$ Stress}

During $\mathrm{NaHCO}_{3}$ stress, $B h A B C$ genes generally exhibited low levels of expression in xylem,

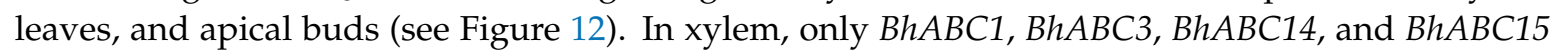
responded to $\mathrm{NaHCO}_{3}$ stress, with $B h A B C 15$ showing downregulated expression during the middle treatment stage, $B h A B C 1$ and $B h A B C 3$ showing upregulation at $6 \mathrm{~h}$, and upregulation observed for $B h A B C 14$ at $72 \mathrm{~h}$. In leaves, regulation of $B h A B C 15$ expression resembled its regulation pattern in xylem, showing a downregulated expression pattern during later treatment stages. $B h A B C 2$ expression was upregulated to a relatively high level at $24 \mathrm{~h}$, whereas $B h A B C 11$ was upregulated during middle and late treatment stages. In apical buds, $B h A B C 2, B h A B C 11, B h A B C 12, B h A B C 14$, and $B h A B C 15$ expression levels were mainly upregulated during middle and later treatment stages. In roots, $B h A B C 1$, $B h A B C 3, B h A B C 6, B h A B C 7, B h A B C 8, B h A B C 9, B h A B C 10, B h A B C 11, B h A B C 14$, and BhABC15 exhibited upregulation during middle and later stages of treatment, with $B h A B C 3, B h A B C 7, B h A B C 9, B h A B C 11$, and $B h A B C 14$ exhibiting significantly increased expression levels at $72 \mathrm{~h}$. No changes in the expression of remaining genes were observed.

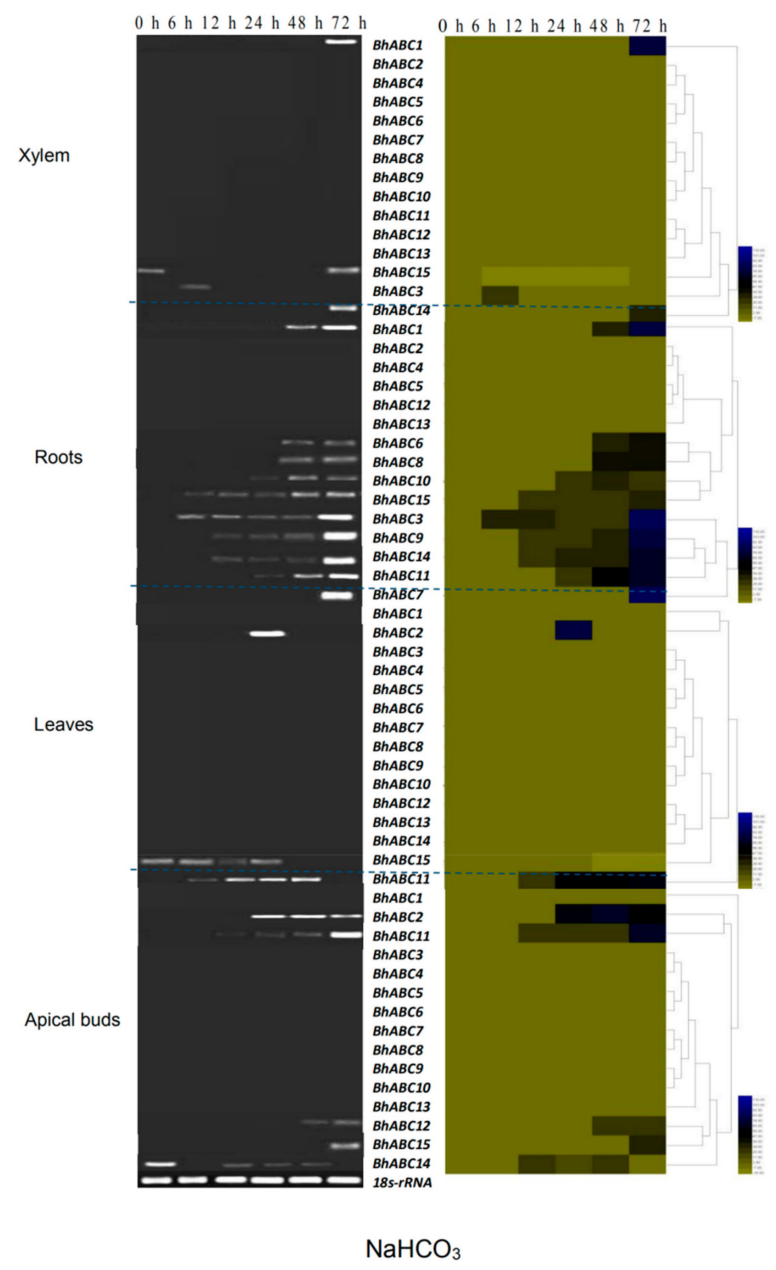

Figure 12. Expression patterns of $B h A B C$ genes in xylem, roots, leaves, and apical buds after $\mathrm{NaHCO}_{3}$ treatment. Heat map showing responses of $B h A B C$ genes to $\mathrm{NaHCO}_{3}$ stress (right); semi-quantitative RT-PCR analysis with 18s RNA internal reference (left).

\subsubsection{Expression of BhABC Genes under Polyethylene Glycol (PEG) stress}

Genes exhibiting altered mRNA expression after plant exposure to PEG stress were mainly BhABC full and half transporters, with altered expression observed in the four tissues studied (see Figure 13). In xylem, $B h A B C 11$ and $B h A B C 15$ showed opposing transcriptional responses of upregulation and 
downregulation, respectively. $B h A B C 1$ and $B h A B C 2$ were downregulated at $6 \mathrm{~h}$ and $48 \mathrm{~h}$, respectively, whereas $B h A B C 6$ and $B h A B C 14$ were upregulated at $72 \mathrm{~h}$. In roots, $B h A B C 11$ was downregulated during the middle treatment stage, whereas $B h A B C 12$ and $B h A B C 15$ were upregulated then downregulated. Meanwhile, $B h A B C 1, B h A B C 2, B h A B C 3, B h A B C 5$, and $B h A B C 14$ genes were upregulated at some time points, whereas no changes in the expression of remaining genes were observed. In leaves, expression levels of $B h A B C 6$ and $B h A B C 15$ were first downregulated then upregulated and expression levels of $B h A B C 1, B h A B C 2, B h A B C 3, B h A B C 5, B h A B C 7, B h A B C 8, B h A B C 11, B h A B C 12$, and $B h A B C 14$ were all upregulated to varying degrees over the course of treatment. No changes in the expression of remaining genes were observed. In apical buds, $B h A B C 11, B h A B C 14$, and $B h A B C 15$ were all upregulated then downregulated, whereas $B h A B C 1, B h A B C 5, B h A B C 6, B h A B C 7, B h A B C 8$, and $B h A B C 12$ were all upregulated, with expression of $B h A B C 12$ peaking during the first PEG treatment stage. No changes in the expression of remaining genes were observed.

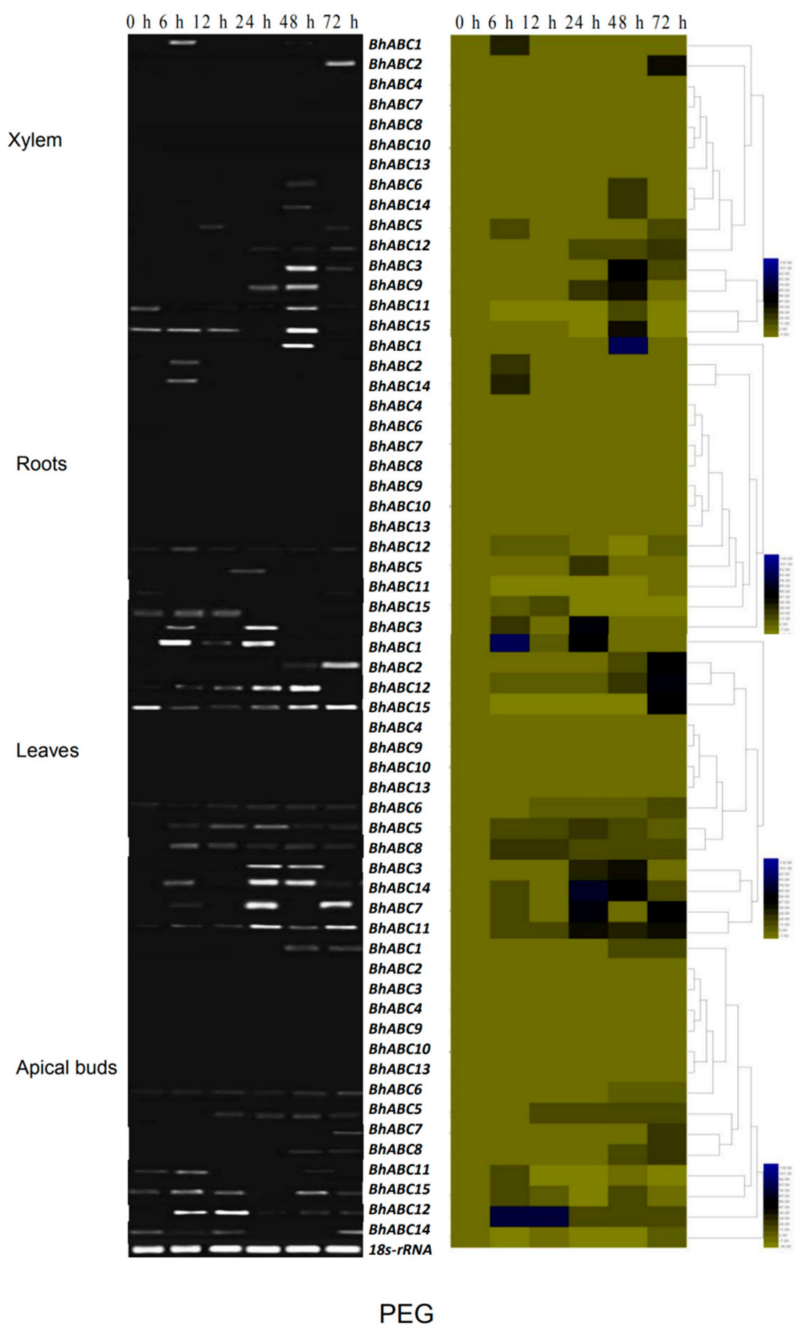

Figure 13. Expression patterns of $B h A B C$ genes in xylem, roots, leaves, and apical buds after PEG treatment. Heat map showing responses of $B h A B C$ genes to PEG (right); semi-quantitative RT-PCR analysis with 18s RNA internal reference (left).

\section{Discussion}

After analyzing physical and chemical properties of 15 BhABC family proteins deduced from transcriptome gene sequences, six BhABC proteins were found to lack transmembrane domains. Subsequent prediction of structural domains and phylogenetic tree analysis revealed six genes coding for soluble $\mathrm{ABC}$ proteins belonging to $\mathrm{ABCF}$ and $\mathrm{ABCI}$ subfamilies. We constructed a preliminary 
phylogenetic tree of BhABC transporter proteins showing that they were distributed among six subfamilies of which several contained subgroups (in parentheses): ABCB (MDR), ABCB (TAP), ABCF, ABCG (PDR), ABCG (WBC), and ABCI. Except for the ABCI subfamily, all other subfamilies were associated with single phylogenetic tree branches (see Figure 2), a result that aligns with previously reported phylogenetic tree results for Arabidopsis and O. sativa ABC transporter proteins [27]. As ABCI proteins contain only a single domain (NBD or auxiliary domain), clustering of members of this subfamily was observed within three distinct phylogenetic tree branches, each closely associated with different subfamilies. The phylogenetic tree shows that protein pairs with greatest homology, namely BhABC7 paired with BhABC11 and BhABC10 paired with BhABC15, exhibited 72.33\% and 92.87\% amino acid sequence similarity, respectively. Comparisons among ABC transporters of B. halophila, A. thaliana, and $O$. sativa demonstrated variability in relative evolutionary distances between proteins. For example, three pairs of close homologs, BhABC8 and OsABCI15, BhABC14 and AtABCG24, and BhABC2 and AtABCI17, exhibited protein sequence similarities of $77.03 \%, 60.59 \%$, and $70.79 \%$, respectively. Greater homology of transporters across subfamilies than among members within a given subfamily may be explained by the functional conservation of structure between protein homologs of herbs and woody plants. However, although the similarity between BhABC3 and OsABCB25 was 65\%, the similarity between BhABC5 homologs of $A$. thaliana and $O$. sativa was only about $50 \%$, suggesting that substantial genetic and functional differences exist between woody and herbaceous plants.

We studied expression characteristics of $B h A B C$ genes in response to exogenous plant hormones and abiotic stressors using semi-quantitative RT-PCR analysis. Of the genes responding to these stimuli, a high proportion belonged to $A B C B$ and $A B C G$ subfamilies, which happen to be the same plant subfamilies currently under study for their suspected roles in exogenous hormone and abiotic stressor responses [4].As $B h A B C$ s are known to exhibit variable expression patterns in response to exogenous hormones, under IAA treatment $B h A B C 11$ ( $A B C B$ subfamily, subgroup $M D R$ ) was upregulated in roots and $B h A B C B$ $(M D R)$ genes $B h A B C 7$ and $B h A B C 11$ were upregulated in leaves and apical buds. At the same time, IAA led to increased gene expression in $B$. halophila roots, leaves, and apical buds of $A B C G$ (PDR) genes $B h A B C 9$ and $B h A B C 15$. In $A$. thaliana, the $A t A B C B 19, A t A B C B 4, A t A B C B 11 / M D R 8, A t A B C B 14 / M D R 12$, and At $A B C B 15 / M D R 13$ genes are involved in auxin transport and regulation [11,12,28], with Indole-3-Butytric acid (IBA ) serving as a storage form of IAA. AtABCG36/PDR8 mediates IBA output and regulates its accumulation in cells, contributing to the regulation of intracellular auxin dynamic balance [29]. We found that treatment with IAA led to increased gene expression in B. halophila roots, leaves, and apical buds of $A B C G$ (PDR) genes BhABC9 and BhABC15. Meanwhile, cytokinins are substances mainly synthesized in root tips which regulate plant growth during water transport from xylem to aboveground plant tissues. The AtABCG14 gene, a member of subgroup $A B C G(W B C)$, is involved in transport of cytokinins in roots [24]. Notably, in $B$. halophila, 6-BA treatment led to upregulated expression of the $A B C G(W B C)$ gene $B h A B C 14$ only in roots, raising speculation that $B h A B C 14$ may share functionality with AtABCG14. Likewise, after ABA treatment, some $B h A B C G$ subfamilies showed upregulated expression patterns in the four tissue types studied here; some of these results align with results of an earlier study demonstrating that stomata of loss-of-function AtABCG40 mutants closed more slowly in response to ABA, resulting in reduced drought tolerance [21]. In addition, another transport protein, AtABCG22, may play a role in ABA signal transduction and ABA biosynthesis [30,31]. Meanwhile, GmPDR12 in soybean (Glycine max) can be expressed rapidly and in large quantities in response to treatment with salicylic acid and its functional derivatives [32]; here, after salicylic acid treatment BhABC15 (PDR) was downregulated in xylem, roots, and leaves. Taken together, the collective results demonstrate that $\mathrm{ABC}$ transporters may have different functions in woody versus herbaceous plants.

When under abiotic stress from cadmium treatment, Arabidopsis seedlings upregulate AtPDR8 (ABCG subfamily) in roots, stems, and other plant tissues [33], whereas ABCB HMT1 protein can improve cadmium tolerance in yeast cells [34,35]. The relationship between ABC transporter proteins and drought resistance has also been studied, with expression of proteins encoded by the $A B C G$ gene subfamily in millet found to vary to differing degrees in response to PEG stress [36]. Moreover, in 
A. thaliana, AtABCG40 (PDR) gene expression relies on the ABA signaling pathway to regulate the rate of stomatal closure, thereby increasing the ability of plants to resist drought [19]. Meanwhile, AtABCG36 helps plants resist drought and salt by reducing the amount of sodium in the plant [37]. Here, $\mathrm{CdCl}_{2}$ treatment resulted in variable changes in expression levels of most $B h A B C G(P D R)$ genes in the four plant tissues studied, with $B h A B C B$ genes mainly downregulated in xylem and roots. During PEG treatment, $A B C G$ gene expression was altered in all four tissues, whereas during $\mathrm{NaCl}$ stress, expression of $A B C G(P D R)$ genes in xylem, leaves, and apical buds were all upregulated.

Under stress, the balance between various hormone levels is disrupted, leading to disturbances in plant growth rhythms and disordered metabolic functions. Therefore, below a certain limit, plants can apparently adjust their physiological functions to compensate for changing hormone levels in order to adapt to adverse environmental conditions. In the abovementioned experiment, all genes that were upregulated in response to $\mathrm{ABA}$ hormone treatment were upregulated in all four tissues in this study. The expression patterns of $\mathrm{BhABC}$ transporters in response to $\mathrm{NaCl}, \mathrm{PEG}, \mathrm{NaHCO}, \mathrm{CdCl}_{2}$, and $\mathrm{ABA}$ treatments suggest that $B h A B C$ genes, which are involved in different abiotic stress responses, may be regulated by ABA-dependent stress signaling pathways.

\section{Conclusions}

The 15 identified $B h A B C$ genes were assigned to four major subfamilies. In the $A B C B$ subfamily were $B h A B C 7$ and $B h A B C 11$ (MDR) and $B h A B C 3$ (TAP); in the $A B C G$ subfamily were $B h A B C 1, B h A B C 9$, $B h A B C 10, B h A B C 12, B h A B C 15$ (PDR), and $B h A B C 14$ (WBC); the $A B C F$ subfamily contained $B h A B C 5$ and $B h A B C 6$; all remaining genes ( $B h A B C 2, B h A B C 4, B h A B C 8$, and $B h A B C 13$ ) were assigned to subfamily $\mathrm{ABCI}$. After treatment with seven exogenous phytohormones and four abiotic stress treatments, it was found that most $A B C B$ and $A B C G$ genes were involved in plant responses to hormones and abiotic stress. We note that molecular mechanisms underlying these responses require further study.

Author Contributions: Conceptualization, J.J., H.L., and L.A.; Formal analysis, L.A., Q.M., M.Y. and F.L.; Software, L.A., J.D., and J.L.; Writing—original draft, L.A.; Writing—review and editing, H.L.

Funding: This work was supported by the Provincial Funding for Major National Science and Technology Projects and Key R\&D Projects in Heilongjiang Province (GX18B027), the Innovation Project of State Key Laboratory of Tree Genetics and Breeding (Northeast Forestry University) (2015B01), and the 111 Project (B16010).

Conflicts of Interest: The authors declare that the research was conducted in the absence of any commercial or financial relationships that could be construed as, or have the appearance of being, a potential conflict of interest.

$\begin{array}{ll}\text { Abbreviations } \\ \text { NBD } & \text { Nucleotide binding domains } \\ \text { TMD } & \text { Transmembrane domains } \\ \text { ORF } & \text { Open reading frame } \\ \text { 6-BA } & \text { 6-Benzylaminopurine } \\ \text { BR } & \text { Brassinolide } \\ \text { GA } 3 & \text { Gibberellin } \\ \text { SA } & \text { Salicylic acid } \\ \text { JA } & \text { Jasmonic acid } \\ \text { IAA } & \text { Indole-3-acetic acid } \\ \text { ABA } & \text { Abscisic acid } \\ \text { PDR } & \text { Pleiotropic drug resistance } \\ \text { WBC } & \text { White-brown complex } \\ \text { ATH } & \text { ABC2 homologue } \\ \text { MDR } & \text { Multidrug resistance protein } \\ \text { TAP } & \text { Transporter associated with antigen processing } \\ \text { ATM } & \text { ABC transporter of the mitochondria } \\ \text { MRP } & \text { Multidrug resistance-associated protein }\end{array}$




\section{References}

1. Glavinas, H.; Krajcsi, P.; Cserepes, J.; Sarkadi, B. The role of ABC transporters in drug resistance, metabolism and toxicity. Curr. Drug Deliv. 2004, 1, 27-42. [CrossRef] [PubMed]

2. Shukla, S.; Wu, C.P.; Ambudkar, S.V. Development of inhibitors of ATP-binding cassette drug transporters-present status and challenges. Expert Opin. Drug Metab. Toxicol. 2008, 4, 205-223. [CrossRef] [PubMed]

3. Beek, J.T.; Guskov, A.; Slotboom, D.J. Structural diversity of ABC transporters. J. Gen. Physiol. 2014, 143, 419-435. [CrossRef] [PubMed]

4. $\quad$ Wang, X.Z.; Sun, W.M.; Ma, Y.F.; Han, E.Q.; Han, L.; Sun, L.P.; Peng, Z.H.; Wang, B.J. Research progress of ABC transporters in Arabidopsis thaliana. Plant. Physiol. 2017, 2, 4-15.

5. Verrier, P.J.; Bird, D.; Burla, B.; Dassa, E.; Forestier, C.; Geisler, M.; Klein, M.; Kolukisaoglu, U.; Lee, Y.; Martinoia, E.; et al. Plant ABC proteins-A unifi ed nomenclature and updated inventory. Trends Plant. Sci. 2008, 13, 151-159. [CrossRef] [PubMed]

6. Kang, J.; Park, J.; Choi, H.; Burla, B.; Kretzschmar, T.; Lee, Y.; Martinoia, E. Plant ABC transporters. In Arabidopsis Book; BioOne: Washington, DC, USA, 2011; Volume 9, p. e0153.

7. Dudle, R.; Hertig, C. Structure of an mdr-like gene from Arabidopsis thatiana. J. Biol. Chem. 1992, 267, 5882-5888.

8. Kougioumoutzi, E.; Cartolano, M.; Canales, C.; Dupré, M.; Bramsiepe, J.; Vlad, D.; Rast, M.; Dello Ioio, R.; Tattersall, A.; Schnittger, A.; et al. SIMPLE LEAF3 encodes a ribosome-associated protein required for leaflet development in Cardamine hirsuta. Plant J. 2013, 73, 533-545. [CrossRef]

9. Navarro-Quiles, C.; Mateo-Bonmati, E.; Micol, J.L. ABCE Proteins: From Molecules to Development. Front. Plant Sci. 2018, 9, 1125. [CrossRef]

10. Maathuis, F.J.; Filatov, V.; Herzyk, P.C.; Krijger, G.B.; Axelsen, K.; Chen, S.; Green, B.J.; Li, Y.; Madagan, K.L.; Sanchez-Fernandez, R.; et al. Transcriptome analysis of root transporters reveals participation of multiple gene families in the response to cation stress. Plant J. 2003, 35, 675-692. [CrossRef]

11. Wu, G.; Carville, J.S.; Spalding, E.P. ABCB19-mediated polar auxin transport modulates Arabidopsis hypocotyl elongation and the endoreplication variant of the cell cycle. Plant J. 2016, 85, 209-218. [CrossRef]

12. Kubeš, M.; Yang, H.; Richter, G.L.; Cheng, Y.; Młodzińska, E.; Wang, X.; Blakeslee, J.J.; Carraro, N.; Petrášek, J.; Zažímalová, E.; et al. The Arabidopsis concentration-dependent infl ux/effl ux transporter ABCB4 regulates cellular auxin levels in the root epidermis. Plant J. 2012, 69, 640-654. [CrossRef] [PubMed]

13. Chen, S.X.; et al. Functional Characterization of AtATM1, AtATM2, and AtATM3, a subfamily of Aradopsis half-molecule ATP-binding cassette transporters implicated in iron homeostasis. J. Bio. Chem. 2001, 282, 21561-21571. [CrossRef] [PubMed]

14. Kim, D.Y.; Bovet, L.; Kushnir, S.; Noh, E.W.; Martinoia, E.; Lee, Y. ArATM3 is invovled in heavy metal resistance in Arabidopsis. Plant Physiol. 2006, 140, 922-932. [CrossRef] [PubMed]

15. Kim, S.; Selote, D.S.; Vatamaniuk, O.K. The N-terminal extension domain of the C. elegans half-molecule ABC transporter, HMT-1, is required for protein-protein interactions and function. PLOS ONE 2010, 5, e12938. [CrossRef] [PubMed]

16. Park, J.; Song, W.Y.; Ko, D.; Eom, Y.; Hansen, T.H.; Schiller, M.; Lee, T.G.; Martinoia, E.; Lee, Y. The phytochelatin transporters AtABCC1 and AtABCC2 mediate tolerance to cadmium and mercury. Plant J. 2012, 69, 278-288. [CrossRef] [PubMed]

17. Song, W.Y.; Park, J.; Mendoza-Cózatl, D.G.; Suter-Grotemeyer, M.; Shim, D.; Hörtensteiner, S.; Geisler, M.; Weder, B.; Rea, P.A.; Rentsch, D.; et al. Arsenic tolerance in Arabidopsis is mediated by two ABCC-type phytochelatin transporters. Proc. Natl. Acad. Sci. USA 2010, 107, 21187-21192. [CrossRef] [PubMed]

18. Brunetti, P.; Zanella, L.; De, P.A.; Di Litta, D.; Cecchetti, V.; Falasca, G.; Barbieri, M.; Altamura, M.M.; Costantino, P.; Cardarelli, M. Cadmium-inducible expression of the ABC-type transporter AtABCC3 increases phytochelatin-mediated cadmium tolerance in Arabidopsis. J. Exp. Bot. 2015, 66, 3815-3829. [CrossRef]

19. Nagy, R.; Grob, H.; Weder, B.; Green, P.; Klein, M.; Frelet-Barrand, A.; Schjoerring, J.K.; Brearley, C.; Martinoia, E. The Arabidopsis ATP-binding cassette protein AtMRP5/AtABCC5 is a high affinity inositol hexakisphosphate transporter involved in guard cell signaling and phytate storage. J. Biol. Chem. 2009, 284, 33614-33622. [CrossRef] 
20. Moons, A. Ospdr9, which encodes a PDR- type ABCtransporter, is induced by heavy metals, hypoxic stress and redox perturbations in rice roots. FEBS Lett. 2003, 553, 370-375. [CrossRef]

21. Kang, J.; Hwang, J.U.; Lee, M.; Kim, Y.Y.; Assmann, S.M.; Martinoia, E.; Lee, Y. PDR-Type ABC Transporter Mediates Cellular Uptake of the Phytohormone Abscisic Acid. Proc. Natl. Acad. Sci. USA 2010, 107, 2355-2360. [CrossRef]

22. Kang, J.; Yim, S.; Choi, H.; Kim, A.; Lee, K.P.; Lopez-Molina, L.; Martinoia, E.; Lee, Y. Abscisic acid transporters cooperate to control seed germination. Nat. Commun. 2015, 6, 8113. [CrossRef] [PubMed]

23. Kuromori, T.; Miyaji, T.; Yabuuchi, H.; Shimizu, H.; Sugimoto, E.; Kamiya, A.; Moriyama, Y.; Shinozaki, K. ABC transporter AtABCG25 is involved in abscisic acid transport and responses. Proc. Natl. Acad Sci USA 2010, 107, 2361-2366. [CrossRef] [PubMed]

24. Ko, D.; Kang, J.; Kiba, T.; Park, J.; Kojima, M.; Do, J.; Kim, K.Y.; Kwon, M.; Endler, A.; Song, W.Y.; et al. Arabidopsis ABCG14 is essential for the root-to-apical bud translocation of cytokinin. Proc. Natl. Acad. Sci. USA 2014, 111, 7150-7155. [CrossRef] [PubMed]

25. Zhang, K.; Novak, O.; Wei, Z.; Gou, M.; Zhang, X.; Yu, Y.; Yang, H.; Cai, Y.; Strnad, M.; Liu, C.J. Arabidopsis ABCG14 protein controls the acropetal translocation of root-synthesized cytokinins. Nat. Commun. 2014, 5, 3247. [CrossRef] [PubMed]

26. Shao, F.J.; Zhang, L.S.; Iain, W.W.; Qiu, D.Y. Transcriptomic Analysis of Betula halophila in Response to Salt Stress. Int. J. Mol. Sci. 2018, 19, 3412.

27. Nguyen, V.N.T.; Moon, S.; Jung, K.H. Genome-wide expression analysis of rice ABC transporter family across spatio-temporal samples and in response to abiotic stresses. J. Plant Physiol. 2014, 171, 1276-1288. [CrossRef] [PubMed]

28. Kaneda, M.; Schuetz, M.; Lin, B.S.P.; Chanis, C.; Hamberger, B.; Western, T.L.; Ehlting, J.; Samuels, A.L. ABC transporters coordinately expressed during lignifi cation of Arabidopsis stems include a set of ABCBs associated with auxin transport. J. Exp. Bot. 2011, 62, 2063-2077. [CrossRef]

29. Strader, L.C.; Bartel, B. The Arabidopsis PLEIOTROPIC DRUG RESISTANCE8/ABCG36 ATP binding cassette transporter modulates sensitivity to the auxin precursor indole-3-butyric acid. Plant Cell 2009, 21, 1992-2007. [CrossRef]

30. Ha, C.V.; Leyva-González, M.A.; Osakabe, Y.; Tran, U.T.; Nishiyama, R.; Watanabe, Y.; Tanaka, M.; Seki, M.; Yamaquchi, S.; Dong, N.V.; et al. Positive regulatory role of strigolactone in plant responses to drought and salt stress. Proc. Natl. Acad. Sci. USA 2014, 11, 851-856. [CrossRef]

31. Merilo, E.; Jalakas, P.; Laanemets, K.; Mohammadi, O.; Hõrak, H.; Kollist, H.; Brosché, M. Abscisic acid transport and homeostasis in the context of stomatal regulation. Mol. Plant 2015, 8, 1321-1333. [CrossRef]

32. Eichhorn, H.; Klinghammer, M.; Becht, P.; Tenhaken, R. Isolation of a novel ABC-transporter gene from soybean induced by salicylic acid. J. Exp. Bot. 2006, 57, 2193-2201. [CrossRef] [PubMed]

33. Kim, D.Y.; Bovet, L.; Maeshima, M.; Martinoia, E.; Lee, Y. The ABC transporter AtPDR8 is a cadmium extrusion pump conferring heav ymetal resistance. Plant J. 2007, 50, 207-218. [CrossRef] [PubMed]

34. Ortiz, D.F.; Kreppel, L.; Speiser, D.M.; Scheel, G. Heavy metal tolerance in the fission yeast requires an ATP-binding cassette- type vacuolar membrane transporter. EMBO J. 1992, 11, 3491-3499. [CrossRef] [PubMed]

35. Ortiz, D.F.; Ruscitti, T.; McCue, K.F.; Ow, D.W. Transport of metal-binding peptides by HMT1, a fission yeast ABC-type vacuolar membrane protein. J. Biol. Chem. 1995, 270, 4721-4728. [CrossRef] [PubMed]

36. Zhang, R.J.; Wang, Z.; Lian, B.Y.; Guo, Z.; Wei, D.; Yu, S.H.; Li, H.Y.; Liu, X.D. Study on the relationship between $\mathrm{ABC}$ transporter genes and drought tolerance in foxtail millet. Shanxi Agric. Univ. 2018, 38, 011.

37. Kim, D.Y.; Jin, J.Y.; Alejandro, S.; Lee, Y. Overexpression of AtABCG36 improves drought and salt stress resistance in Arabidopsis. Physiol. Plant. 2010, 139, 170-180. [CrossRef] [PubMed]

(C) 2019 by the authors. Licensee MDPI, Basel, Switzerland. This article is an open access article distributed under the terms and conditions of the Creative Commons Attribution (CC BY) license (http://creativecommons.org/licenses/by/4.0/). 